\title{
NFAT restricts osteochondroma formation from entheseal progenitors
}

\author{
Xianpeng Ge, ${ }^{1,2}$ Kelly Tsang, ${ }^{1}$ Lizhi He, ${ }^{3}$ Roberto A. Garcia, ${ }^{4}$ Joerg Ermann, ${ }^{1}$ Fumitaka Mizoguchi, ${ }^{1}$ \\ Minjie Zhang, ${ }^{5}$ Bin Zhou, ${ }^{6}$ Bin Zhou, ${ }^{7}$ and Antonios O. Aliprantis ${ }^{1}$ \\ 'Department of Medicine, Division of Rheumatology, Immunology and Allergy, Brigham and Women's Hospital and \\ Harvard Medical School, Boston, Massachusetts, USA. ${ }^{2}$ Central Laboratory, Peking University School and Hospital of \\ Stomatology, Beijing, China. ${ }^{3}$ Department of Biological Chemistry and Molecular Pharmacology, Harvard Medical School, \\ Boston, Massachusetts, USA. ${ }^{4}$ Department of Pathology, Bone and Soft Tissue Pathology Division, Icahn School of \\ Medicine at Mount Sinai, New York, USA. ${ }^{5}$ Orthopaedic Research Laboratories, Boston Children's Hospital and Harvard \\ Medical School, Boston, Massachusetts, USA. ${ }^{6}$ Department of Genetics, Pediatrics, and Medicine (Cardiology), Albert \\ Einstein College of Medicine of Yeshiva University, New York, USA. 'Key Laboratory of Nutrition and Metabolism, Institute \\ for Nutritional Sciences, Shanghai Institutes for Biological Sciences, Chinese Academy of Sciences, Shanghai, China.
}

\begin{abstract}
Osteochondromas are common benign osteocartilaginous tumors in children and adolescents characterized by cartilage-capped bony projections on the surface of bones. These tumors often cause pain, deformity, fracture, and musculoskeletal dysfunction, and they occasionally undergo malignant transformation. The pathogenesis of osteochondromas remains poorly understood. Here, we demonstrate that nuclear factor of activated T cells $\mathrm{c} 1$ and $\mathrm{c} 2$ (NFATc1 and NFATc2) suppress osteochondromagenesis through individual and combinatorial mechanisms. In mice, conditional deletion of NFATc1 in mesenchymal limb progenitors, Scleraxis-expressing (Scx-expressing) tendoligamentous cells, or postnatally in Aggrecan-expressing cells resulted in osteochondroma formation at entheses, the insertion sites of ligaments and tendons onto bone. Combinatorial deletion of NFATc1 and NFATc2 gave rise to larger and more numerous osteochondromas in inverse proportion to gene dosage. A population of entheseal NFATc1- and Aggrecan-expressing cells was identified as the osteochondroma precursor, previously believed to be growth plate derived or perichondrium derived. Mechanistically, we show that NFATc1 restricts the proliferation and chondrogenesis of osteochondroma precursors. In contrast, NFATc2 preferentially inhibits chondrocyte hypertrophy and osteogenesis. Together, our findings identify and characterize a mechanism of osteochondroma formation and suggest that regulating NFAT activity is a new therapeutic approach for skeletal diseases characterized by defective or exaggerated osteochondral growth.
\end{abstract}

Conflict of interest: The authors have declared that no conflict of interest exists.

Submitted: December 28, 2015 Accepted: February 29, 2016 Published: April 7, 2016

Reference information: JCI Insight. 2016;1(4):e86254. doi:10.1172/jci.insight.86254.

\section{Introduction}

Osteochondromas, the most common primary skeletal tumor (1-3), are cartilage-capped bony projections containing marrow cavities continuous with that of the underlying bone (4). These lesions may occur at a single site in the sporadic form (termed solitary osteochondroma [SO]) or throughout the skeleton in a hereditary form (termed hereditary multiple osteochondromas [HMO]). Most osteochondromas develop at the metaphyses of long bones in close proximity to the growth plate. However, they also appear on the epiphyses or diaphyses of long bones and on flat bones and vertebrae $(5,6)$. Extraskeletal osteochondromas without a direct attachment to bone may also arise, commonly associated with tendons, ligaments, joint capsules, or synovial membranes (7-10). Osteochondromas can cause a variety of secondary problems, including growth retardation, deformities, fracture, chronic pain, compression of nerve and blood vessels, degenerative joint disease, and psychological concerns. Moreover, in $1 \%$ of SO and $2 \%-5 \%$ of HMO cases, the exostoses undergo malignant transformation $(3,6)$.

Current understanding of the pathogenesis of osteochondromas is limited. Heterozygous loss-of-function mutations in the exostosin 1 or 2 (EXT1 or EXT2) genes are found in about $80 \%-90 \%$ of HMO and in some SO patients (11-16). However, EXT haploinsufficiency is insufficient for osteochondroma 
A

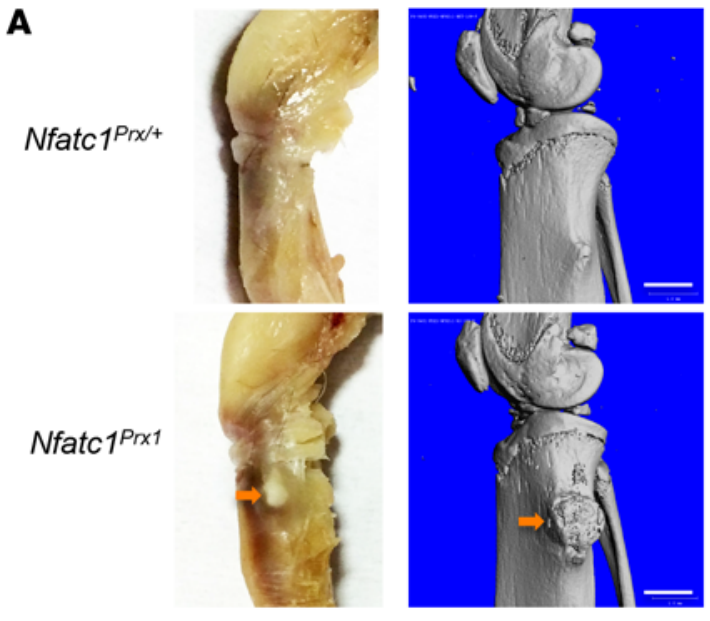

B

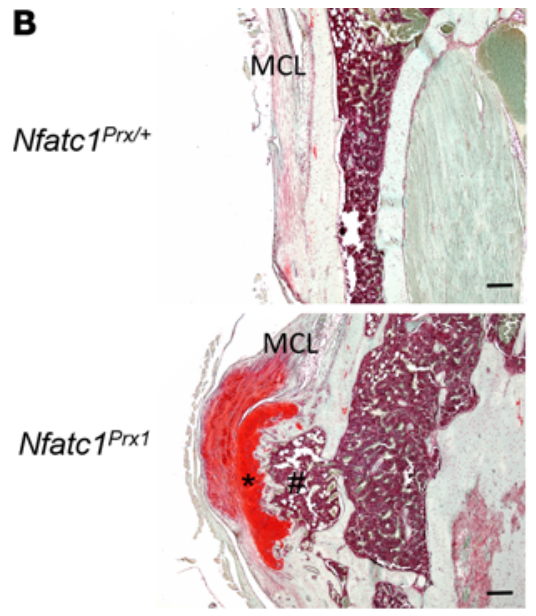

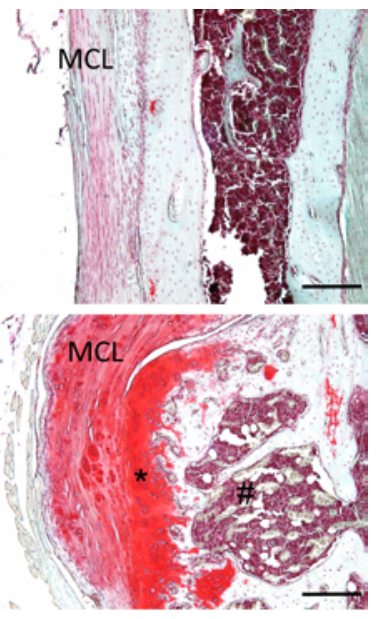

C

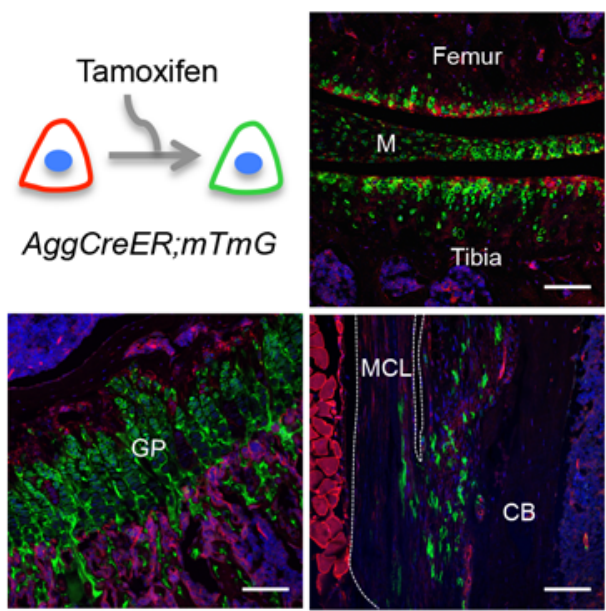

D
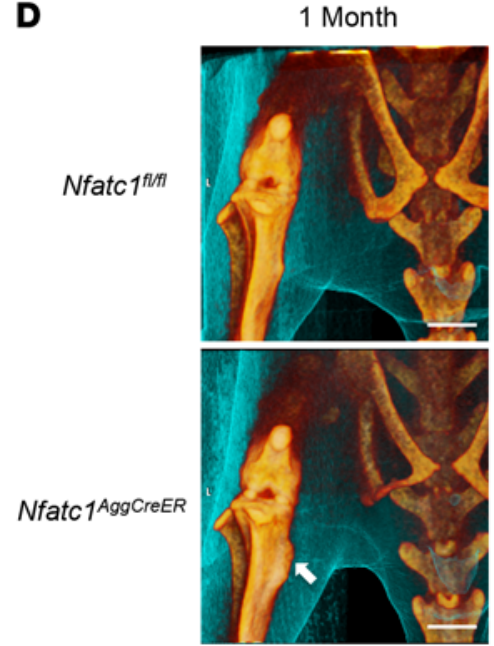

3 Months
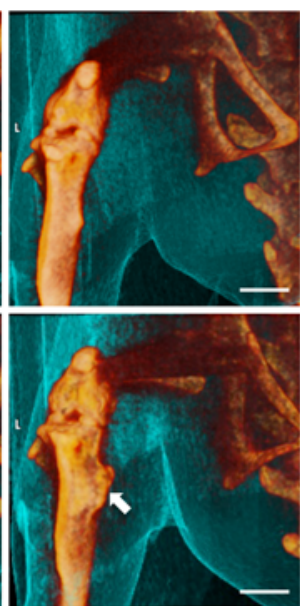

3 Months

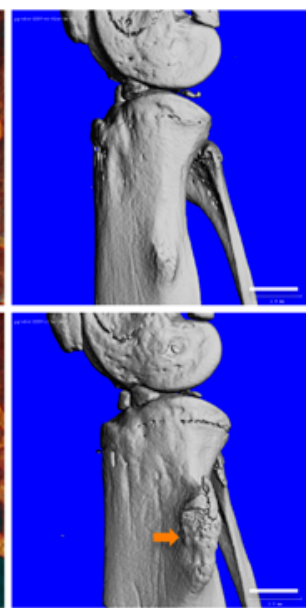

Figure 1. Ablation of nuclear factor of activated T cells c1 (NFATc1) in limb mesenchymal progenitors and postnatal Aggrecan-expressing cells causes osteochondromas at entheseal sites. (A) Gross (left panels) and 3-D $\mu \mathrm{CT}$ images (right panels) showing ectopic osteocartilaginous outgrowths (arrows) at the proximal tibia of 16 -week-old $N$ fatc $1^{\text {Prx }}$ mice. Scale bars: $1.0 \mathrm{~mm}$. Images are representative of 8 mice per genotype. (B) Safranin $0 /$ fast green stain of coronal sections of mouse knee joints showing abnormal outgrowths in $N f a t c 1^{P r x}$ mice located at the tibial medial collateral ligament (MCL) enthesis and displaying features of osteochondromas, including a cartilaginous cap $\left(^{*}\right)$ and the marrow cavity (\#), continuous with that of underlying bone. Scale bars: $100 \mu \mathrm{m}$. (C) Illustration showing tamoxifen-induced Cre-mediated recombination of the mTmG reporter allele in Aggrecan ${ }^{+}$cells from Aggrecan-CreERT2; Rosa26-mTmG $\mathrm{C}^{f /+}$ (AggCreER;mTmG) mice leading to membrane GFP expression (upper left panel). GFP fluorescence in the growth plate (GP, lower left panel), articular cartilage, meniscus (M, upper right panel), and a subset of cells at the tibial MCL enthesis (lower right panel) in AggCreER;mTmG mice 1 month after tamoxifen administration. Scale bars: $100 \mu \mathrm{m}$. CB, cortical bone. Images are representative of 5 mice. (D) In vivo (left and middle panels) and ex vivo (right panels) 3-D $\mu \mathrm{CT}$ images showing osteochondroma formation at the tibial MCL enthesis (arrows) 1 and 3 months after tamoxifen pulse of an 8-week-old Nfatc7 ${ }^{A g g c r e E R}$ mouse. Scale bars: $5.0 \mathrm{~mm}$ (left and middle panels), $1.0 \mathrm{~mm}$ (right panels). Images are representative of 10 mice per genotype.

formation $(3,11)$. Although loss of heterozygosity can be a potential reason for the disparity $(17-19)$, an alternative mechanism to EXT mutations probably exists, especially for HMO and SO patients lacking EXT gene disruptions $(3,11,20)$. In addition, the cell of origin of osteochondromas remains unresolved (16). Candidates for the cellular origin include growth plate chondrocytes and cells in the periosteum or perichondrium (Groove of Ranvier) (16,19). Further studies are needed to clarify where these common bone tumors originate.

The nuclear factor of activated T cells (NFAT) signaling axis is a vertebrate-specific pathway important for a diverse array of functions (21). Accumulating studies implicate NFATs in many aspects of tumorigenesis, including carcinogenesis, cell proliferation, metastasis, and drug resistance (21-24). In the skeletal system, germ-line deletion of Nfatc2 in mice caused ectopic cartilage formation around synovial joints and the chondrocytes displayed neoplastic changes (25). Furthermore, chromosomal translocation between NFATc1 or NFATc2 gene and the Ewing sarcoma breakpoint region 1 (EWSR1) gene have been identi- 
fied in human hemangioma of the bone and Ewing sarcoma, respectively $(26,27)$. These studies suggest that NFATs may be essential for normal skeletal homeostasis and that abnormalities of this pathway can lead to tumorigenesis. Recently, we reported that cartilage-specific ablation of Nfatc1 by Collagen2-Cre in $\mathrm{Nfatc2}^{-/-}$mice leads to early onset, aggressive osteoarthritis (OA) affecting multiple joints. However, single deletion of $\mathrm{Nfatcl}$ in chondrocytes failed to cause articular cartilage pathology or increased susceptibility to posttraumatic OA $(28,29)$. Thus, the role of NFATc1 in chondrocyte biology is unresolved. Here, we provide evidence linking NFAT deficiency with osteochondroma formation and identify how NFATc1 and NFATc2 differentially regulate cell proliferation and osteochondral differentiation, respectively, to suppress osteochondroma formation from entheseal progenitors.

\section{Results}

NFATc1 is an osteochondroma suppressor gene. To identify the role of NFATc1 in joint and cartilage biology, we deleted $N f a t c 1$ in mouse limb mesenchymal progenitors using the Prx1-cre strain (Prx 1-Cre; Nfatc ${ }^{f l / f l}$, hereafter $\left.N f a t c 1^{P r x}\right)$. Gross images and microcomputed tomography $(\mu \mathrm{CT})$ analysis showed that 16-week-old $N f a t c 1^{P x x l}$ mice - but neither Prx1-Cre; $N f a t c 1^{f /+}$ (hereafter $N f a t c 1^{P r x l /+}$ ) nor $N f a t c 1^{f l f l}$ controls - developed abnormal osteocartilaginous outgrowths at the proximal tibia (Figure 1A). These lesions mapped to the insertion sites of the medial collateral ligament (MCL) or patellar ligament (PL) and demonstrated the typical features of osteochondromas, including a cartilaginous cap and a BM cavity continuous with that of underlying bone (Figure 1B and Supplemental Figure 1A; supplemental material available online with this article; doi:10.1172/jci.insight.86254DS1). In addition, these mice also developed abnormal bony growths around knee joints. These bony abnormalities were composed of ectopic cartilage undergoing endochondral ossification inside the periarticular knee ligaments (Supplemental Figure 1B), which are reminiscent of extraskeletal osteochondromas. Thus, NFATc1 restricts spontaneous osteochondroma formation.

Next, we sought to narrow the cell type in which the NFATc1 deficiency results in osteochondromas and to determine whether post-natal $N f a t c 1$ gene deletion can recapitulate the phenotype of $N f a t c 1^{\text {Prx }}$ mice. An analysis of Aggrecan expression with the tamoxifen-inducible AggrecanCreERT2;Rosa26-mTmG $G^{f /+}$ (hereafter $A g g C r e E R ; m T m G$ ) mice demonstrated that, in addition to the growth plate, articular cartilage, and meniscus, Aggrecan is also expressed in a population of cells at the tibial MCL enthesis (Figure 1C and Supplemental Figure 1, C and D). To determine whether these Aggrecan-expressing cells with Nfatcl deficiency are responsible for osteochondroma formation at the ligamentous entheses, the AggrecanCreERT2;Nfatc $1^{\text {fl/fl }}$ (hereafter $N$ fatc $1^{A g g C r e E R}$ ) strain was generated and received tamoxifen at 8 weeks of age. In vivo and ex vivo $\mu \mathrm{CT}$ analyses demonstrated that, within 1 month of tamoxifen administration, Nfatc1 deletion driven by the Aggrecan promoter resulted in osteochondromas at the MCL entheses, which increased in size with age (Figure 1D).

NFAT gene dosage determines the penetrance and severity of osteochondromas. Functional redundancy between NFAT family members has been reported in many organ systems $(30,31)$. Our previous study indicated that NFATc1 acts cooperatively with NFATc2, but not NFATc3, to repress spontaneous OA in mice (28). To determine whether NFATc1 and NFATc2 are redundant in repressing osteochondroma formation, we intercrossed the $\mathrm{Nfatc1}^{f / / f l}, \mathrm{Nfatc2}^{-{ }^{--}}$, and $\mathrm{AggCreER}$ strains to generate mice with all possible genotypes. Tamoxifen was administrated at 8 or 12 weeks of age. Within 1 month, $N f a t c 1^{A g g C r e E R} N f a t c 2^{+/-}$mice displayed osteochondroma formation at the tibial MCL insertion (Figure 2, A and B). $\mathrm{Nfatc1}^{\mathrm{AggCreER}} \mathrm{Nfatc2}^{-/-}$mice developed osteochondromas at the proximal tibia with higher penetrance and more mature bone within the osteochondroma (Figure 2A). Three months after tamoxifen administration, the osteochondromas in $\mathrm{Nfatc}^{\text {AggCreER }} \mathrm{Nfatc}^{+/-}$ mice were in the process of endochondral ossification (Figure $2 \mathrm{C}$ ), while $N f a t c 1^{A g g C r e E R} N f a t c 2^{-/-}$mice displayed clear osteochondroma-like exostoses characterized by cartilage-capped bony protrusions continuous with the underlying bone (Figure 2C). Notably, $N$ fatc $1^{f l f f} N f a t c 2^{-/-}$mice, which only lack $N f a t c 2$, also developed osteochondroma-like outgrowths at the proximal tibia at this time point (Figure 2C). However, these growths displayed only faint proteoglycan staining, suggesting that they form by direct, rather than endochondral, ossification. Moreover, the osteochondromas in Nfatc1-deficient mice were all sessile, while some of those in Nfatc2 $\mathrm{KO}$ mice displayed a radiographic feature of pedunculated osteochondromas. This suggests that the osteochondromas arising from each gene deletion form via different mechanisms. In addition to accelerated progression of osteochondromas and $100 \%$ penetrance, more $\mathrm{Nfatc}^{\mathrm{Agg} C \mathrm{CrER}} \mathrm{Nfatc2^{-/- }}$ mice developed bilateral osteochondromas (10/13) at the tibial MCL enthesis compared with $N f a t c 1^{\text {AggCreER }} N f a t c 2^{+/-}(5 / 10)$ and $N$ fatc $f^{f / f l} N f a t c 2^{-/-}(2 / 11)$ mice (Supplemental Table 1). Furthermore, the penetrance of osteochondromas 
A
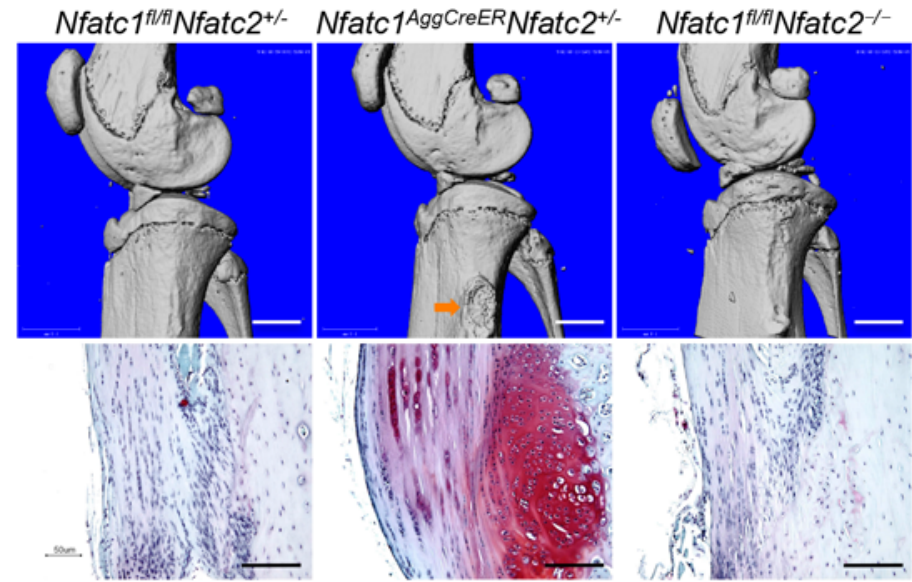

Nfatc1 ${ }^{\text {AggCreER }}$ Nfatc2 $^{-1}$
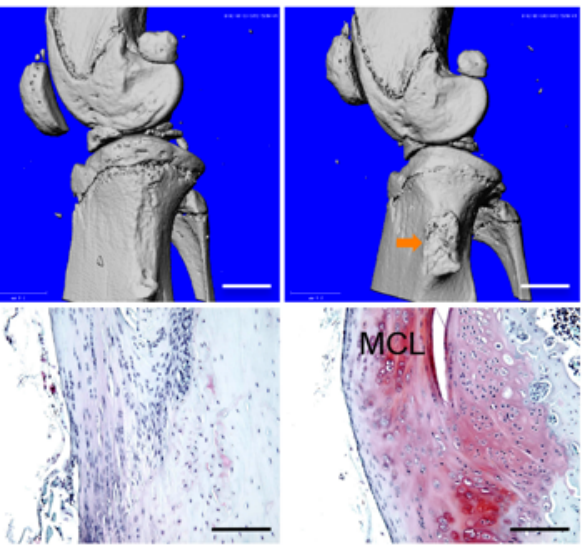

C
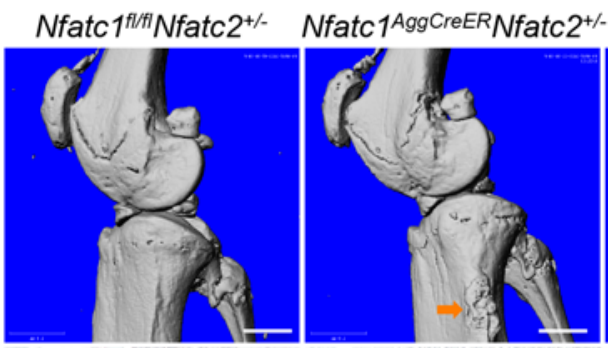

Nfatc1 $1^{\text {flifl }} \mathrm{Nfatc}^{-1-}$
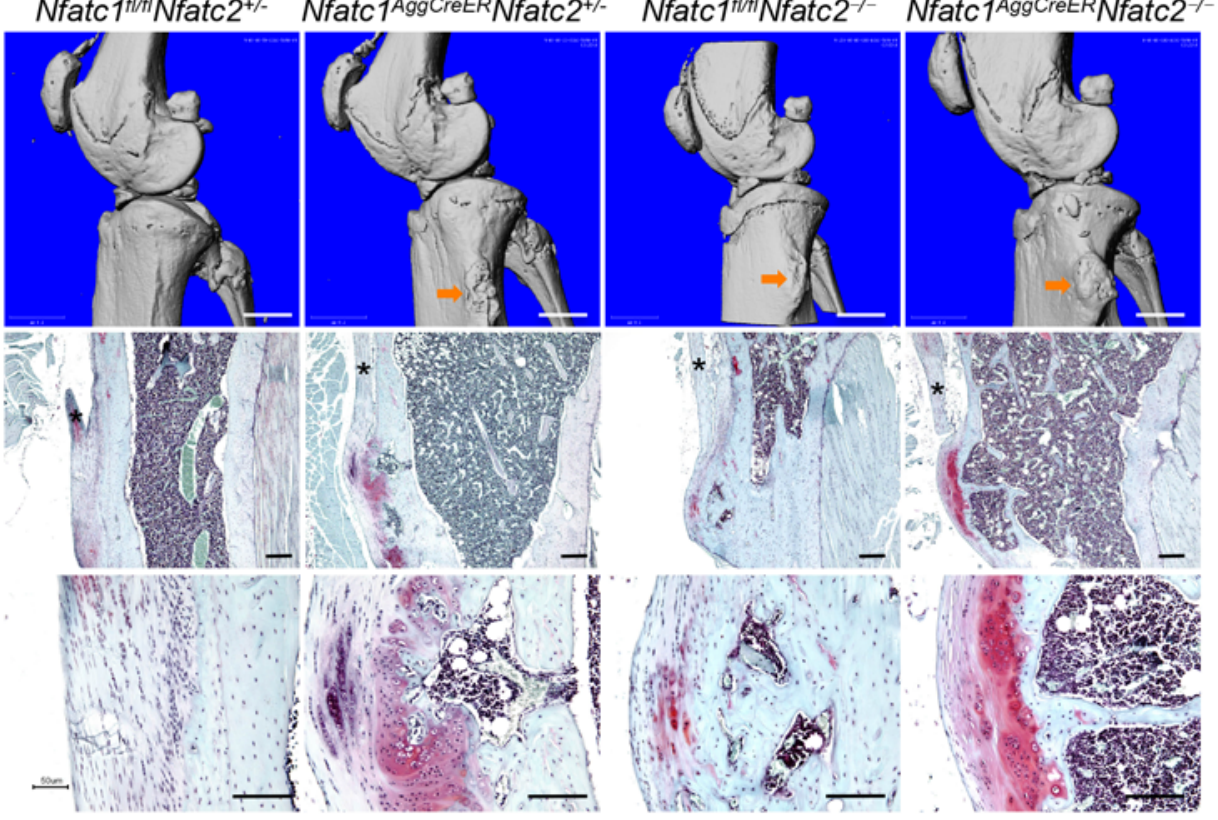

D Gene dosage of Nfatc1 and Nfatc2

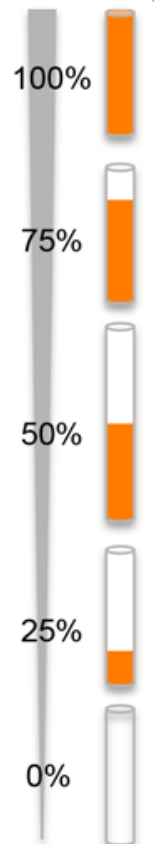

$$
\begin{array}{r}
\text { of Nfatc1 and Nfatc2 } \\
\text { Nfatc } 1 / 1 / 7 \\
n=10 \\
\text { Aggrecan-CreERT2 } \\
n=6
\end{array}
$$

Nfatc1 1 AggCreER/+

$n=10$
Nfatc1 ${ }^{\text {fliff }}$ Nfatc $2^{+/}$
$n=10$

Nfatc1 ${ }^{\text {AggCreER }}$ $n=10$

Nfatc $1^{\text {flffi }} \mathrm{Nfatc}^{-/-}$

$n=11$

Nfatc1 ${ }^{\text {AggCreER/+}}{ }^{-}$fatc $^{+/-}$

$n=10$

Nfatc1 ${ }^{\text {AggCreER Nfatc }} 2^{+/-}$

$n=10$
Nfatc1 1 AggCreER/+Nfatc $2 \%$

$n=10$

Nfatc1 ${ }^{\text {AggCreER Nfatc2- }}$

$n=14$

$$
n=14
$$
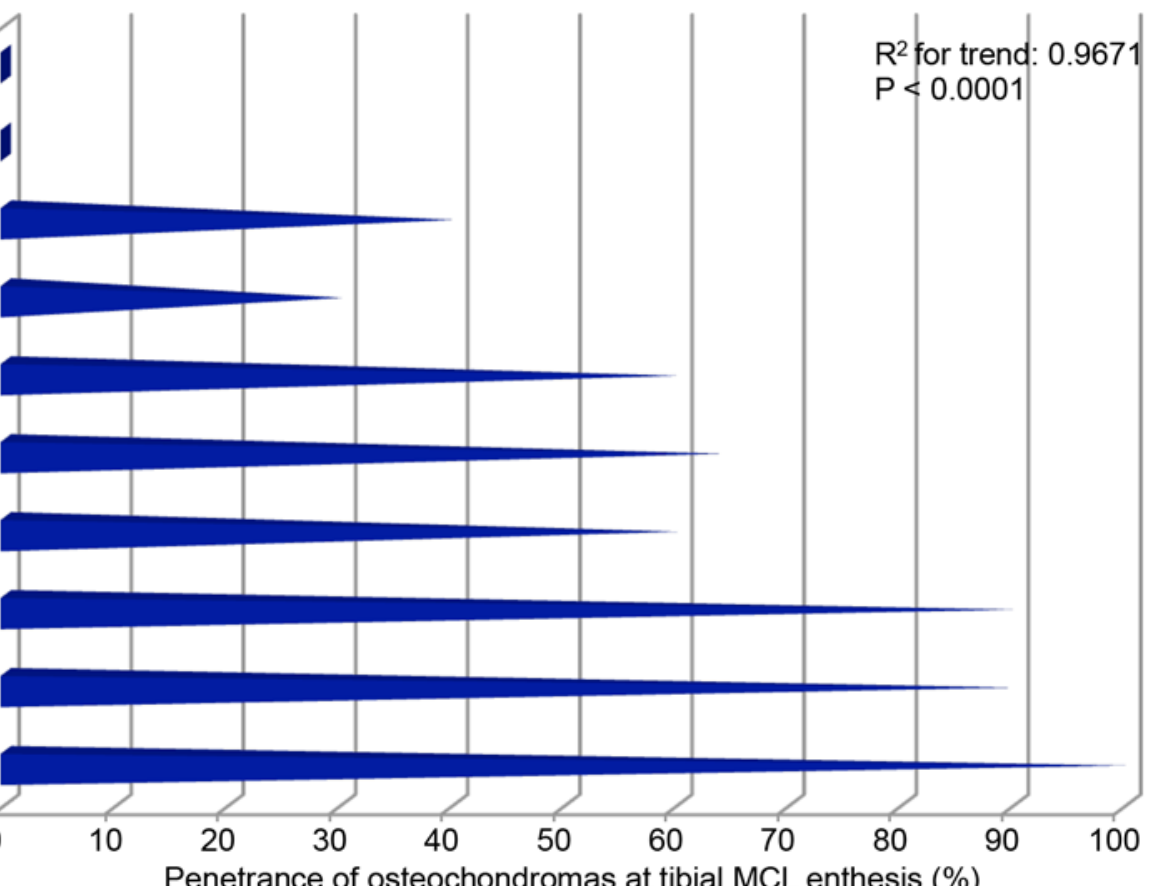

Penetrance of osteochondromas at tibial MCL enthesis (\%) 
Figure 2. Nuclear factor of activated T cells (NFAT) gene dosage determines the severity of osteochondromas. (A) One month after tamoxifen pulse in 8- or 12-week-old mice, representative $\mu \mathrm{CT}$ images and safranin $0 /$ fast green (SF) staining showing osteochondroma formation at the tibial medial

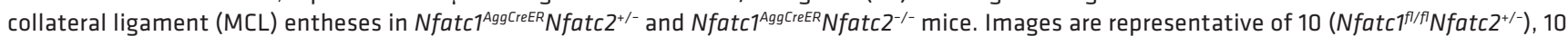

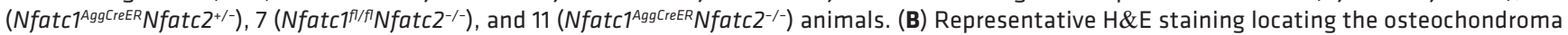

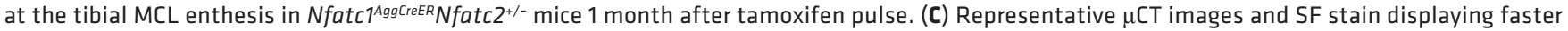

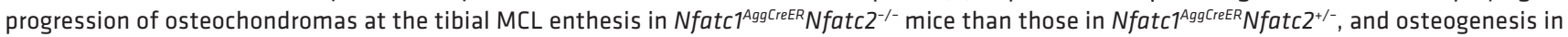

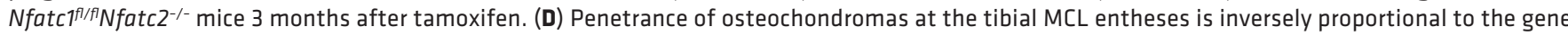
dosage of $N f a t c 1$ and $N f a t c 2$ three months after tamoxifen pulse in 8- or 12-week-old mice. In cases where the Nfatc2 genotype is not noted, the mice were $\mathrm{Nfatc2}^{+/+}$. Linear regression analysis was performed as indicated. ${ }^{*}$ in $\mathbf{B}$ and $\mathbf{C}$ represent MCL. Scale bars: $1.0 \mathrm{~mm}$ (A and $\mathbf{C}$, upper panels), $100 \mu \mathrm{m}$ (A, lower panels; B; and C, middle and lower panels).

at the proximal tibia was inversely related to the gene dosage of $N f a t c 1$ and $N f a t c 2$ (Figure 2D). When tamoxifen was administered to $N f a t c 1^{A g g C r e E R} N f a t c 2^{+/-}$and $N f a t c 1^{A g g C r e E R} N f a t c 2^{-/-}$mice 2 weeks after birth, the animals developed progressive osteochondroma growth with age versus $N f a t c 1^{f l / f l} N f a t c 2^{+/-}$and $N f a t c 1^{f l / f l} \mathrm{Nfatc2}^{2^{--}}$mice (Figure 3). Compared with $N f a t c 1^{A g g C r e E R} N f a t c 2^{+/-}$and $N f a t c 1^{A g g C r e E R} N f a t c 2^{-1-}$ mice that received tamoxifen at 12 weeks of age, those treated at 2 weeks of age developed larger osteochondromas (Figure $2 \mathrm{C}$ and Figure 3). This suggests that the timing of Nfatcl deletion contributes to osteochondroma severity. Taken together, these data indicate functional redundancy between NFATc1 and NFATc2 with regard to suppression of osteochondroma formation. In addition, deletion of NFATc1 and NFATc2 appear to differentially affect osteochondroma formation, with loss of the former resulting in accelerated endochondral growth and loss of the latter enhancing ossification.

Although the tibial MCL enthesis was the site where osteochondromas most commonly formed in mice with NFAT deficiency, these tumors also developed at other locations. In hip joints, $N$ fatc $1^{\text {Agg CreER }} \mathrm{Nfatc2}^{-1-}$ mice displayed more osteochondromas both 1 and 3 months after tamoxifen administration compared with $N f a t c 1^{f l / f l} N f a t c 2^{+/-}, N f a t c 1^{A g g C r e E R} N f a t c 2^{+/-}$, and $N f a t c 1^{f l / f l} N f a t c 2^{-/-}$mice (Figure 4, A and B). Histological analysis showed that these osteochondromas were located at the entheses or associated with tendons, ligaments, or joint capsules around hip joints (Figure 4B). Osteochondromas were also found at other sites where ligaments, tendons, and/or the joint capsule insert onto bone: the tibial enthesis of the PL, the distal and proximal femurs, the proximal humerus, the scapula, and the shoulder joint (Figure 4, C-G). However, we found neither osteochondromas on the hands and feet nor enchondromas in NFAT-deficient mice, which differentiate our model from metachondromatosis, another genetic disease characterized by widespread cartilage tumors $(32,33)$.

A cellular origin of osteochondromas: entheseal mesenchymal progenitors. To identify the cell of origin for osteochondromas in NFAT-deficient mice, we performed lineage-tracing studies using $N f a t c 1^{A g g r e E R} \mathrm{Nfatc}^{-1-}$, Rosa26- $m T m G^{f /+}$ (hereafter $D K O-m T m G$ ) mice. Sections of knee joints from these animals showed that the majority of cells in osteochondromas at the tibial MCL enthesis were $\mathrm{GFP}^{+}$, although cells lacking evidence of Cre-mediated recombination $\left(\mathrm{TdTomato}^{+}\right.$) were also integrated into the tumor (Figure 5A). We reasoned that cells expressing both $N f a t c 1$ and Aggrecan in the MCL ligament, its enthesis, the perichondrium/periosteum of the proximal tibia, or the growth plate represent the primary cellular origin for the osteochondromas observed in NFAT-deficient mice. To identify this cell subpopulation, we used Cre-recombinase reporter mice to overlap the cellular expression of Aggrecan (AggCreER;mTmG mice) with that of Nfatc1 (Nfatc1Cre; Rosa26- $m$ TmG $G^{f /+}$ and Nfatc1CreERT2;Rosa26-RFP ${ }^{f /+}$ mice, hereafter Nfatc1Cre; $m$ TmG and Nfatc1CreER;RFP, respectively). Most growth plate cells expressed GFP in AggCreER; $m$ TmG mice 1 month after tamoxifen (Figure 1C) but were negative for GFP or RFP in Nfatc1Cre; $m$ TmG or Nfatc1CreER;RFP mice, respectively (Figure $5, \mathrm{~B}$ and C). Conversely, cells in the perichondrium/periosteum were $\mathrm{GFP}^{+}$and $\mathrm{RFP}^{+}$in $N$ fatclCre;mTmG and Nfatc1CreER;RFP mice, respectively (Figure 5, B and D) but were GFP- in both AggCreER;mTmG and DKO$m T m G$ mice (Figure 5E). Control animals ( $m T m G$ with Nfatc1-Cre) did not show any GFP labeling (Supplemental Figure 2A). Thus, neither growth plate cells nor perichondrial/periosteal cells are likely to contribute to osteochondroma formation. In contrast, Aggrecan and Nfatc1 were both expressed at the tibial MCL enthesis (Figure 1C and Figure 5, B and D), where most osteochondromas originated. By overlaying the expression patterns of Aggrecan and $\mathrm{Nfatc1}$ near the osteochondroma origin, we conclude that a population of cells in the MCL enthesis contributes to osteochondromas in NFAT-deficient mice (Figure 5F).

Entheses are believed to contain multipotent progenitors that give rise to both ligamentocytes/tenocytes and chondrocytes, which establish ligament/tendon-bone junctions during development (34). Flow cytometry was used to isolate $\mathrm{GFP}^{+}$cells from the tibial MCL enthesis of AggCreER;mTmG mice 1 week 

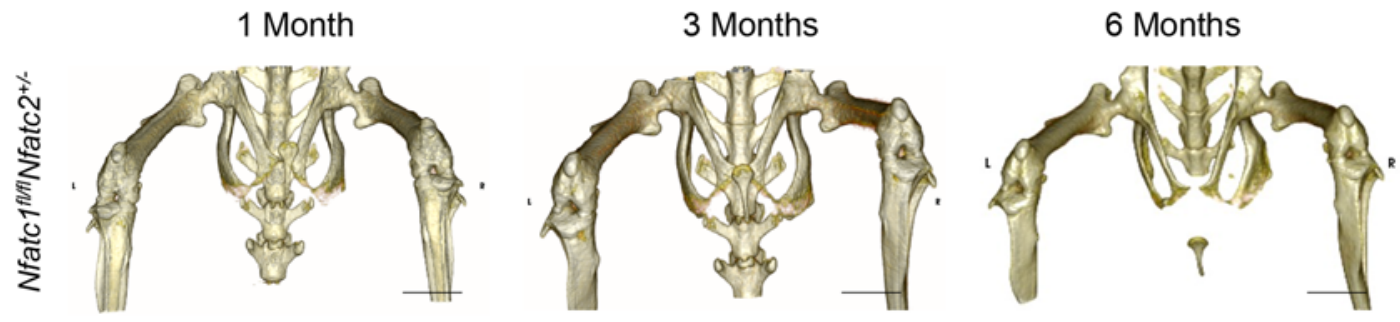

6 Months
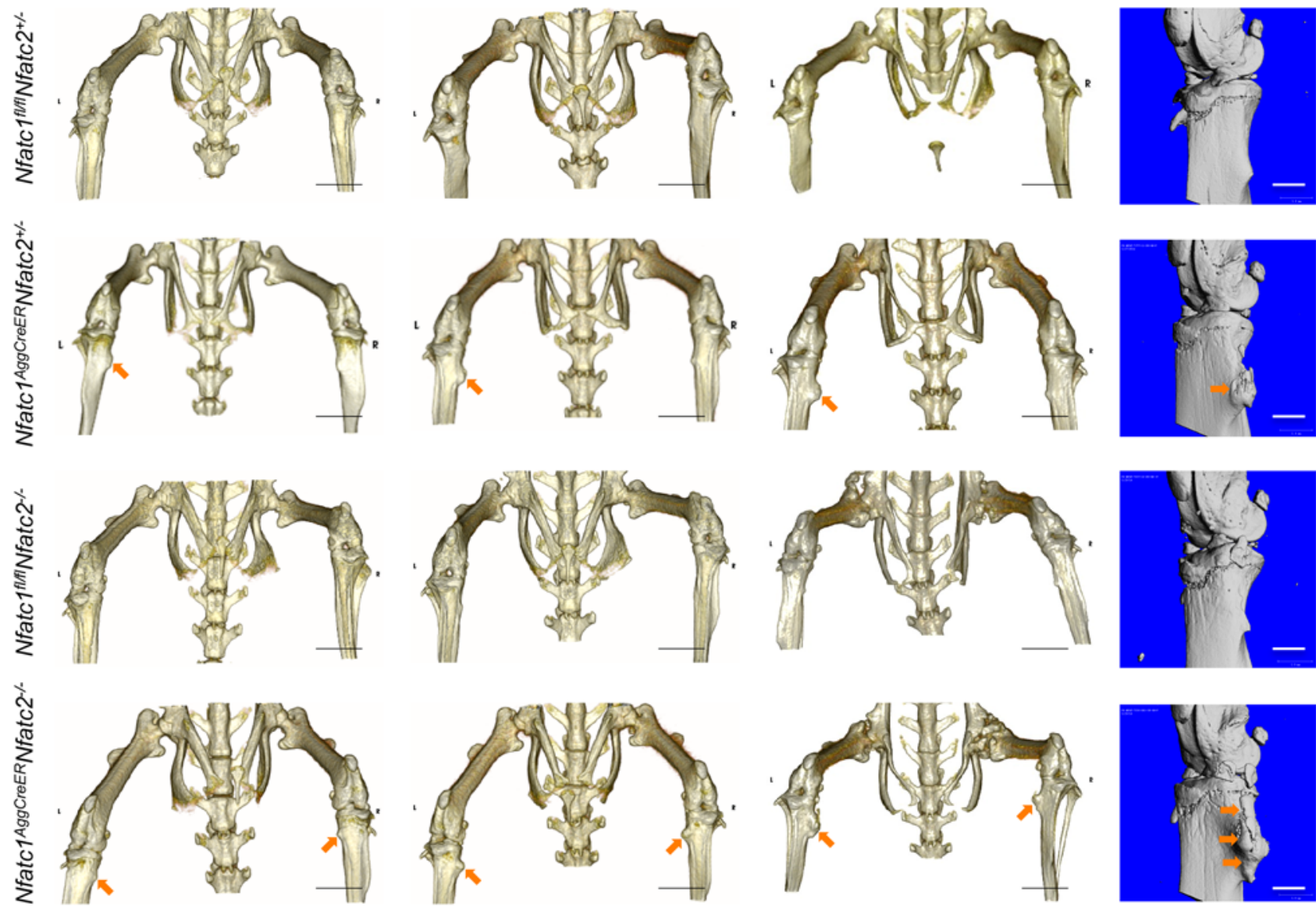

Figure 3. Timing of nuclear factors of activated T cells (NFAT) deletion affects the severity of osteochondromas. In vivo and ex vivo $\mu$ CT images showing pro-

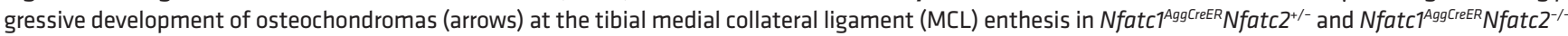

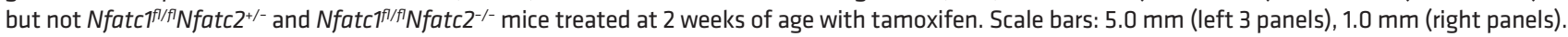
Images are representative of 4 or 5 mice per genotype.

after tamoxifen (Supplemental Figure 2B). Colony formation assay showed that these $\mathrm{GFP}^{+}$cells were clonogenic, and approximately $25 \%-30 \%$ cells formed adherent cell colonies after 10 days of culture (Supplemental Figure 2, C and D). These colonies were heterogeneous in size and cell density, potentially reflecting differences in cell proliferation rates. Under the appropriate lineage-specific differentiation conditions in vitro, these $\mathrm{GFP}^{+}$cells formed adipocytes, osteoblasts, or chondrocytes (Figure 5, G-I). Furthermore, these $\mathrm{GFP}^{+}$cells expressed the mesenchymal progenitor markers Sca1, CD90, and CD44 (35), but not the hematopoietic marker CD45 or endothelial cell marker CD31 (Figure 5J). Taken together, these data indicate that Aggrecan-expressing cells at the entheses are multilineage stromal progenitors and represent a cellular origin of osteochondromas.

Ablation of NFATc1 in Scx-expressing progenitors. Entheseal progenitor cells express the transcriptional factor Scx during development (34). Interestingly, the Aggrecan-expressing entheseal progenitors also expressed a high level of $S c x$ (Supplemental Figure 2E). To determine whether ablation of $N f a t c 1$ in $S c x$-expressing cells can recapitulate osteochondroma formation, $N f a t c 1^{S c x}$ mice were generated. $\mu \mathrm{CT}$ analysis showed that $N f a t c 1^{s c x}$ mice developed osteochondromas at the tibial MCL enthesis (Supplemental Figure 3A). Nfatc1 ${ }^{\text {Scx- }}$

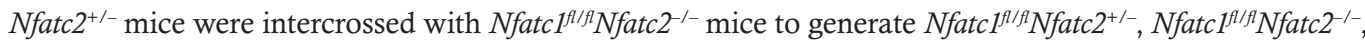
$N f a t c 1^{s c x} N f a t c 2^{+/-}$, and $N f a t c 1^{s c x} N f a t c 2^{-/-}$mice. $N f a t c 1^{s c x} N f a t c 2^{+/-}$mice developed larger and more numerous osteochondromas compared with $\mathrm{Nfatcl}^{\mathrm{AggCreER}} \mathrm{Nfatc2^{+/- }}$ mice (Figure 6, A and B; Supplemental Figure 3B; and Table 1). Notably, the $N f a t c 1^{S c x} \mathrm{Nfatc2}^{-/-}$mice displayed multiple features of $\operatorname{HMO}(36,37)$, including multiple osteochondromas throughout the skeleton, short stature, deformity, and subluxation or dislocation of elbow and ankle joints (Figure 6, A-E, and Supplemental Figure 3, C-H). Moreover, gait assess- 
A
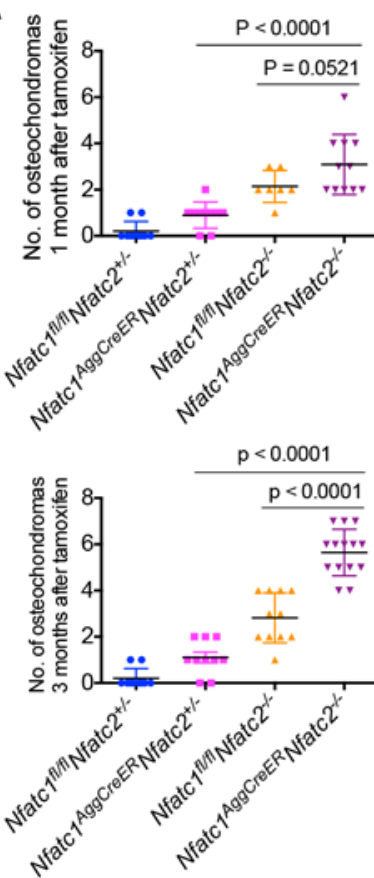

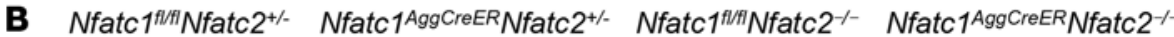
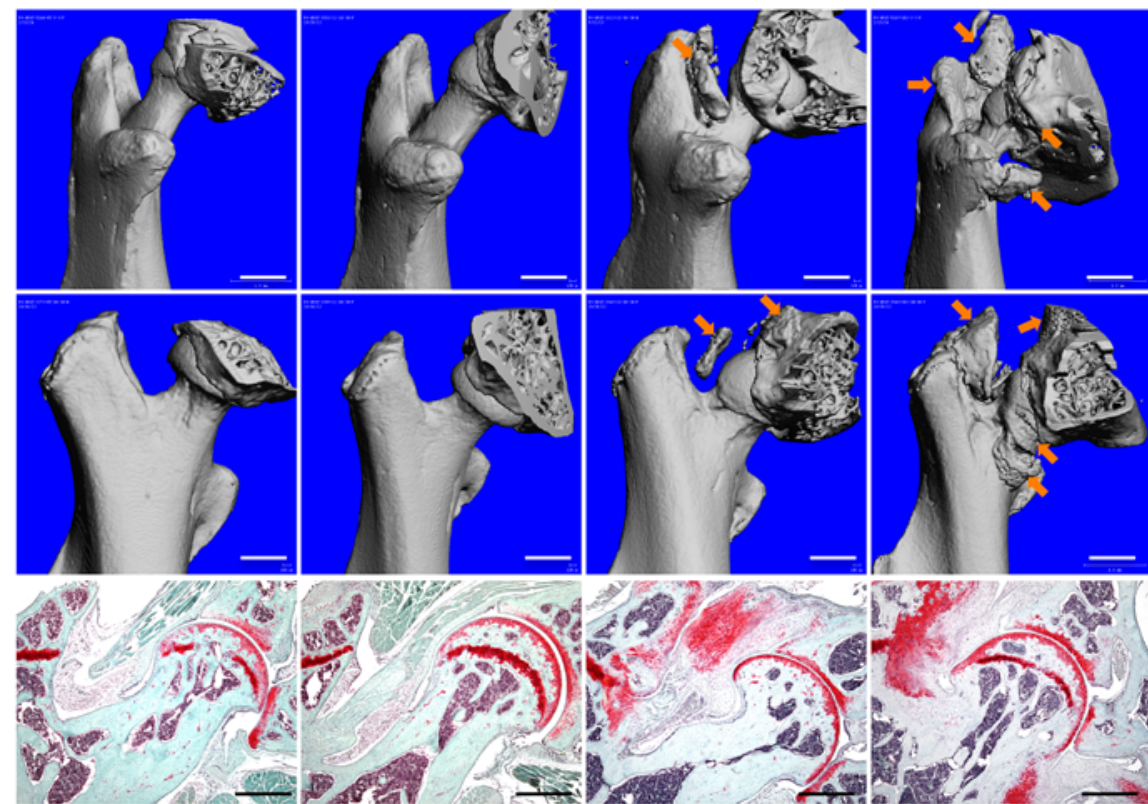

C
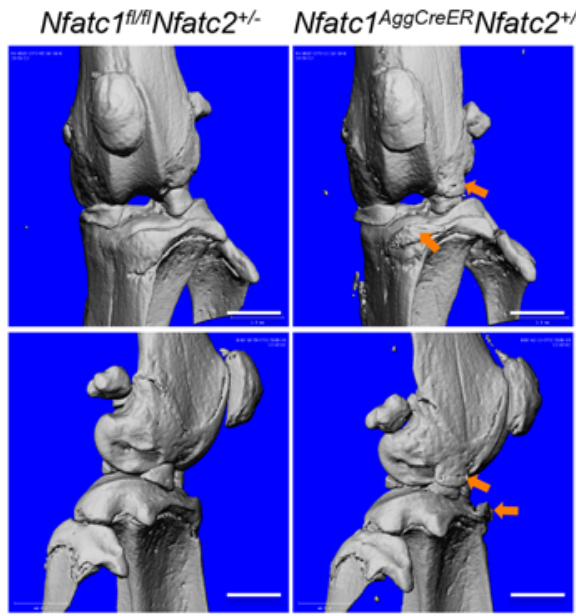

E

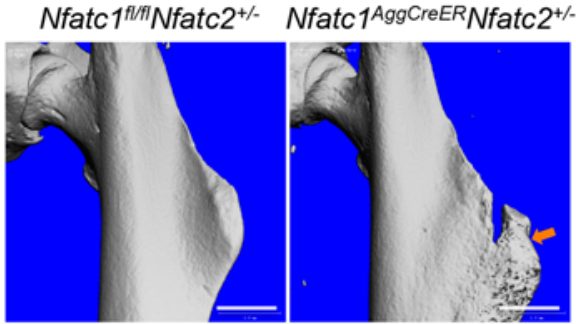

G

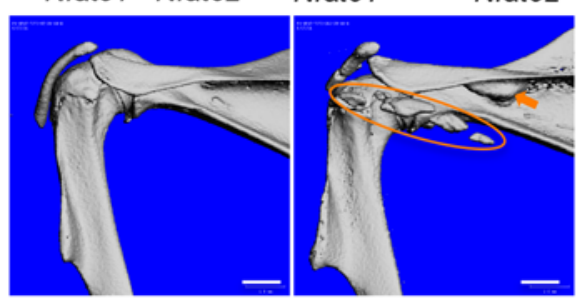

Nfatc1 AggCreERNfatc2 ${ }^{-}$
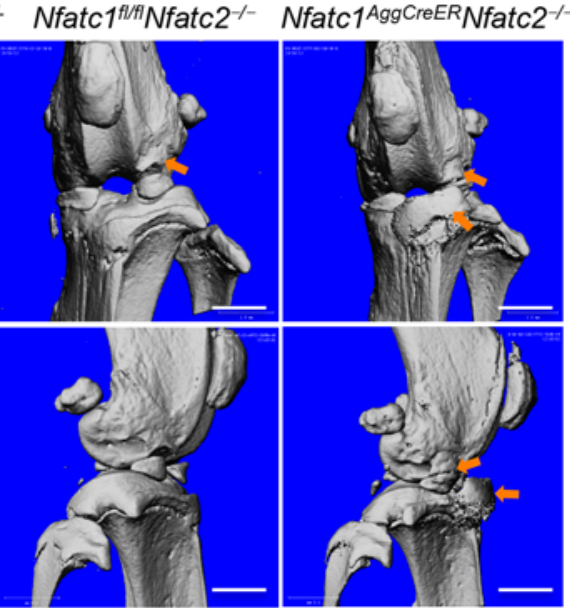

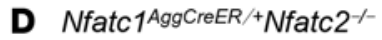

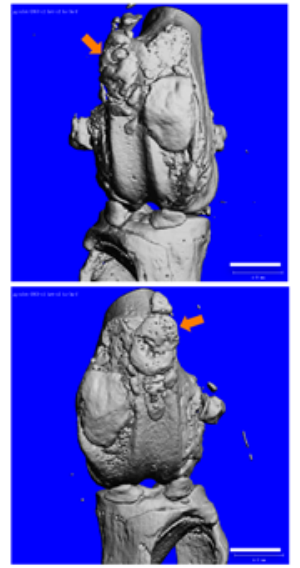

Nfatc $1^{\mathrm{II} / \mathrm{N}} \mathrm{Nfatc2}^{+/-}$
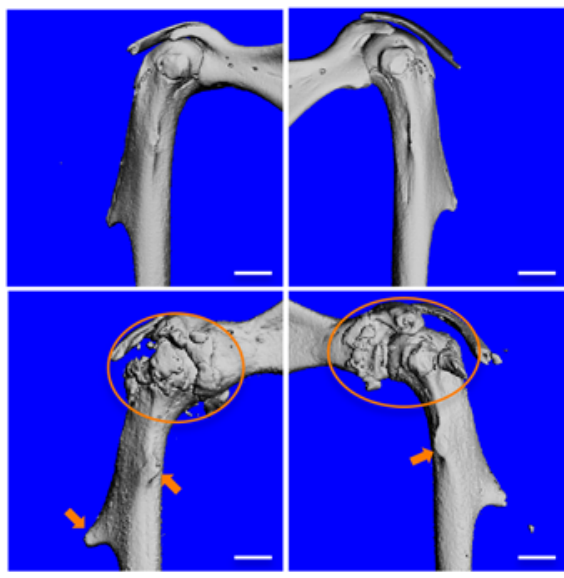

Nfatc1 ${ }^{\text {Agg CreER }} \mathrm{Nfatc2}^{-\prime}$

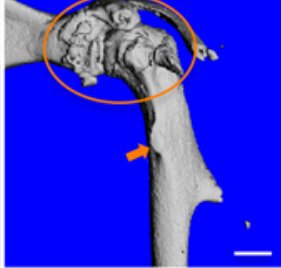


Figure 4. Osteochondroma formation at other locations after combinatorial deletion of nuclear factor of activated T cells c1 and c2 (Nfatc1 and

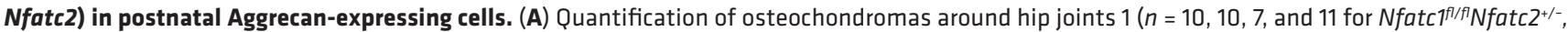

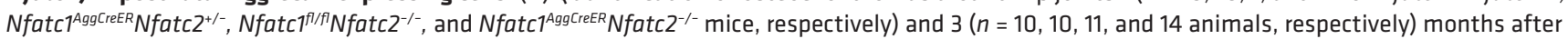
tamoxifen pulse in 8- or 12-week-old mice. One-way ANOVA followed by Tukey's test were performed. (B) Ex vivo $\mu \mathrm{CT}$ images and safranin 0/fast greenstained histology of the hip joints of mice with the indicated genotypes 3 months after tamoxifen administration to 8- or 12-week-old mice. Arrows, osteochondromas. (C) Representative $\mu \mathrm{CT}$ images showing osteochondroma formation at the epiphyses of knee joints (arrows). (D and E) Representative $\mu \mathrm{CT}$ images of osteochondroma formation at distal and proximal femurs (arrows). (F and $\mathbf{G}$ ) Representative $\mu \mathrm{CT}$ images of osteochondromas around shoulder joins (F, circles), at the proximal humerus (F, arrows), and at the scapula (G). Scale bars: $1.0 \mathrm{~mm}$ (except in $\mathbf{B}$, lower panels, $100 \mu \mathrm{m})$.

ment was performed, since patients with HMO often suffer from musculoskeletal dysfunction $(38,39)$. This analysis revealed a significant reduction of contact intensity, stride length, and time of single stance in

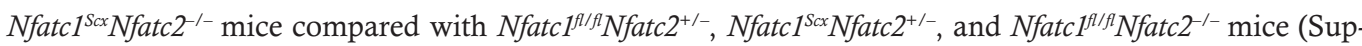
plemental Figure 4, A-D). Restricted hind limb abduction was also observed in the $N f a t c 1^{S c x} N f a t c 2^{-/-}$mice (Supplemental Figure 4E), suggesting a restriction in hip range of motion. Taken together, these data provide further support that entheseal progenitors are the cellular origin of osteochondromas and that the $N \mathrm{fatc}^{\mathrm{Scx}} \mathrm{Nfatc2}^{--}$strain recapitulates the radiographic and functional manifestations of human HMO.

NFATC1 and NFATC2 repress osteochondromagenesis through complimentary mechanisms. To determine the cellular and molecular mechanism of osteochondroma formation after deletion of Nfatc1 and/or Nfatc2, we intercrossed the mice with combinatorial deletion of $N f a t c 1$ and $N f a t c 2$ with $m T m G$ reporter mice to

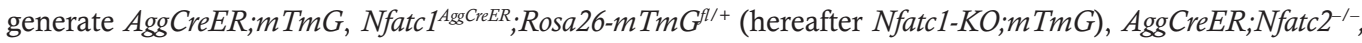
Rosa26- $m T m G^{f /+}$ (hereafter Nfatc2-KO; $m T m G$ ), and $D K O ; m T m G$ mice, and we isolated $\mathrm{GFP}^{+}$cells from the tibial MCL enthesis 1 week after tamoxifen administration (Figure 7A). Of the 4 strains, ex vivo-cultured $\mathrm{GFP}^{+}$cells from Nfatc1-KO;mTmG mice displayed the highest proliferation (Figure 7, B and C). Accordingly, more cells stained positively for proliferating cell nuclear antigen (PCNA), a marker of cell proliferation, at the tibial MCL enthesis of $N f a t c 1^{A g g C r e E R} N f a t c 2^{+/-}$mice compared with $N f a t c 1^{f l / f l} N f a t c 2^{+/-}$mice (Figure 7D). Notably, the $\mathrm{GFP}^{+}$cells from $N f a t c 2-K O ; m T m G$ and $D K O ; m T m G$ mice did not proliferate more than cells from AggCreER;mTmG mice, even though these mice also developed osteochondromas. To confirm and extend these results, $N f a t c 1$ and/or Nfatc2 were deleted in the chondroprogenitor cell line ATDC5 (40) with CRISPR/CAS9 technology (Supplemental Figure 5, A and B). When cultured under nondifferentiating conditions, both CRISPR-Nfatc1 and CRISPR-Nfatc2 ATDC5 lines showed increased proliferation, and a synergistic effect of deleting both genes in CRISPR-DKO ATDC5 cells was observed (Figure 7E). Intriguingly, after 2 weeks of micromass culture in chondrogenic media, the CRISPR-Nfatc 2 and CRISPR-DKO ATDC5 cells displayed less proliferation than CRISPR-Nfatc1 cells (Figure 7F), similar to what was observed in primary cells isolated from Nfatc2-KO;mTmG and $D K O ; m T m G$ mice (Figure 7, B and C). Micromass diameter was larger in CRISPR-Nfatcl and CRISPR-DKO cells compared with CRISPR-Vector and CRISPR-Nfatc2 cells (Figure 7G). This result parallels the progressive growth of osteochondromas at the MCL insertion, which is greater in $N f a t c 1^{A g g C r e E R}$ and $N f a t c 1^{A g g C r e E R} N f a t c 2^{-1-}$ mice compared with WT and $N f a t c f^{f l / f} N{f a t c 2^{-/}}^{-1}$ mice (Figure 3). In contrast, overexpression of a constitutively active form of NFATc1 (caNFATc1) in ATDC5 cells inhibited cell proliferation by blocking G1/S phase progression (Supplemental Figure 5, C-F). Consistent with this effect, ATDC5 cells expressing caNFATc1 displayed upregulation of the cell cycle inhibitor $p 21$ and downregulation of the regulator of cell growth $c-M y c$ (Figure $7 \mathrm{H}$ ). In contrast, ATDC5 cells lacking Nfatc1 and Nfatc2 displayed the opposite effect on $p 21$ and $c-M y c$ expression (Figure 7I). Taken together, these results indicate an important role of NFATc1 in restricting proliferation or maintaining quiescence of entheseal mesenchymal progenitors.

Since endochondral ossification is the typical mechanism for osteochondroma formation, progenitor cells likely differentiate into both chondrocytes and osteoblasts. To determine whether Nfatc1 and Nfatc2 regulate the differentiation of progenitors into one or both of these lineages, gene expression was examined in ATDC5 cells lacking either transcription factor. Cells were cultured under chondrogenic conditions as the formation of chondrocytes is the first step of the endochondral process. In the absence of Nfatc1, cells expressed higher levels of chondrogenic markers Col2a1 and Col10a1 after a 2-week micromass culture (Figure 8A). In contrast, cells lacking $N f a t c 2$ expressed higher levels of hypertrophic chondrocyte and osteogenic markers (Mmp13, Ibsp) (Figure 8B). A similar gene expression pattern of these chondrogenic and osteogenic markers was found in the $\mathrm{GFP}^{+}$cells isolated from $N f a t c 1-K O ; m T m G$ and $N f a t c 2-K O ; m T m G$ mice and cultured for 1 week ex vivo (Figure 8, C and D). In addition, the $\mathrm{GFP}^{+}$cells from $N f a t c 2-K O ; m T m G$ mice also expressed higher levels of osteogenic markers Tnsalp and Colla1 (Figure 8E). Cells from either 
A

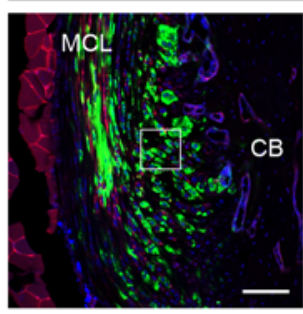

C

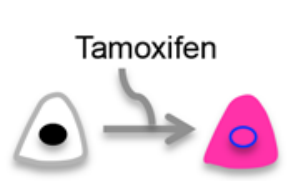

Nfatc1CreER;RFP
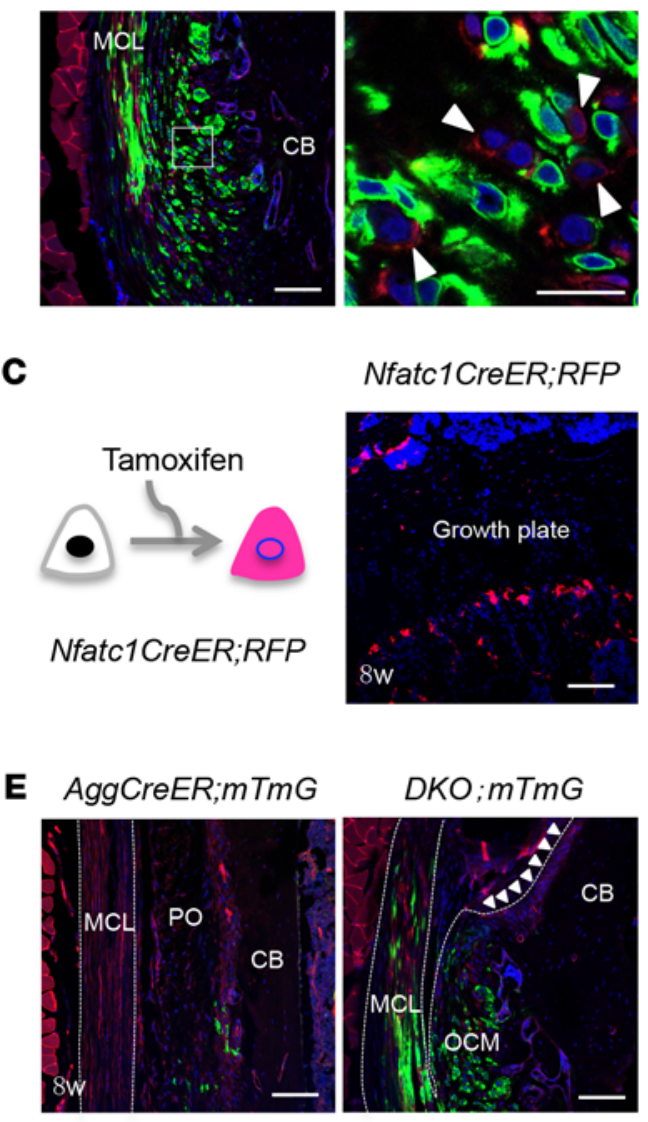

Nfatc1CreER;RFP

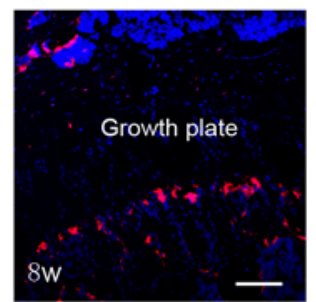

B

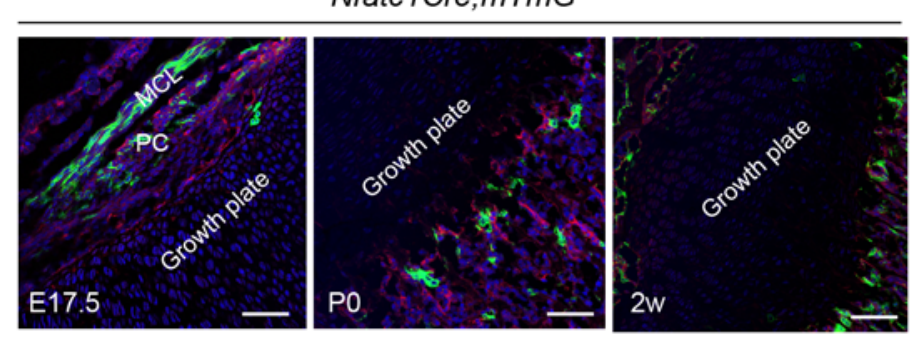

D

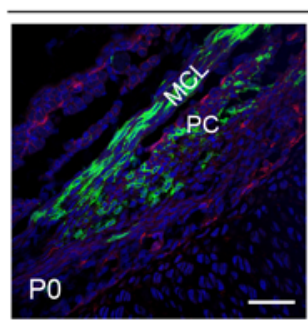

Nfatc1Cre;mTmG

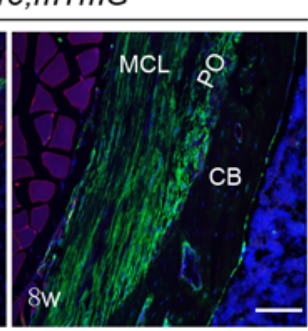

Nfatc1CreER;RFP

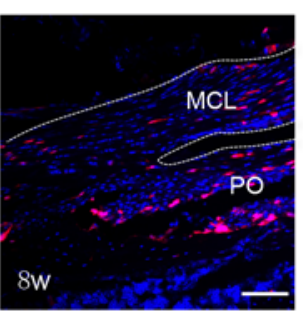

F

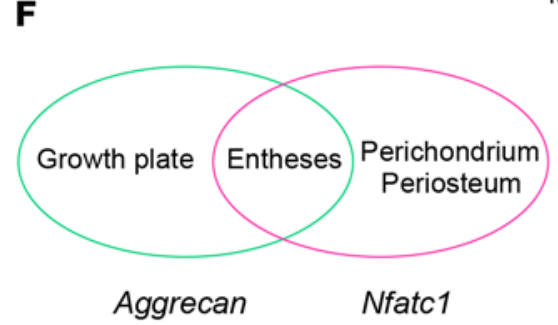

Aggrecan

MCL

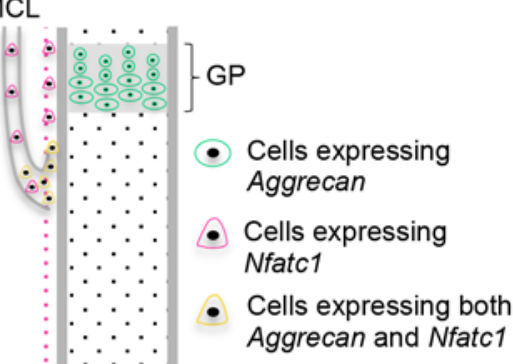

G

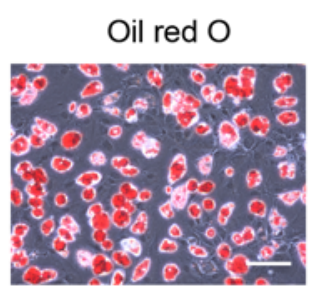

H

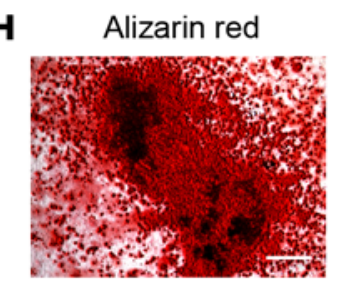

J

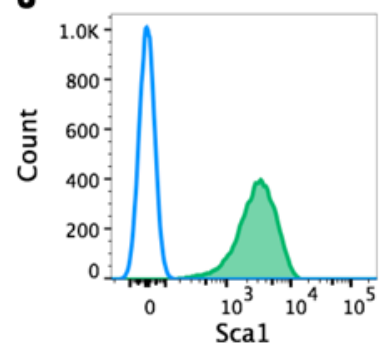

Fabp 4
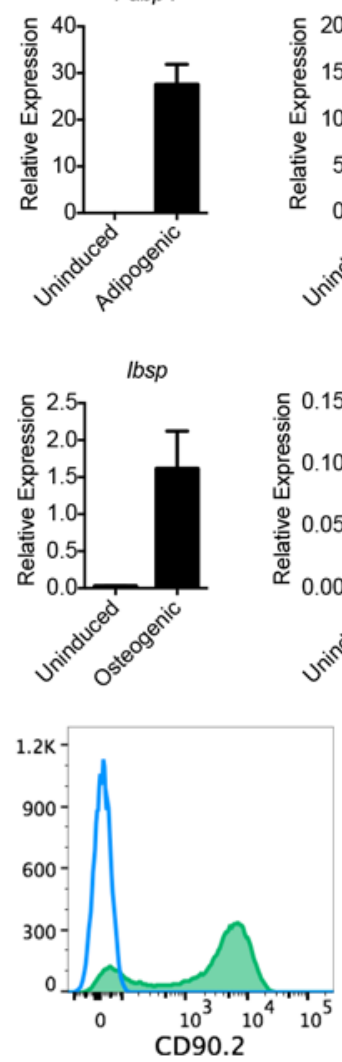

$L p l$

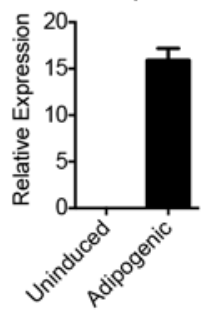

$S p 7$
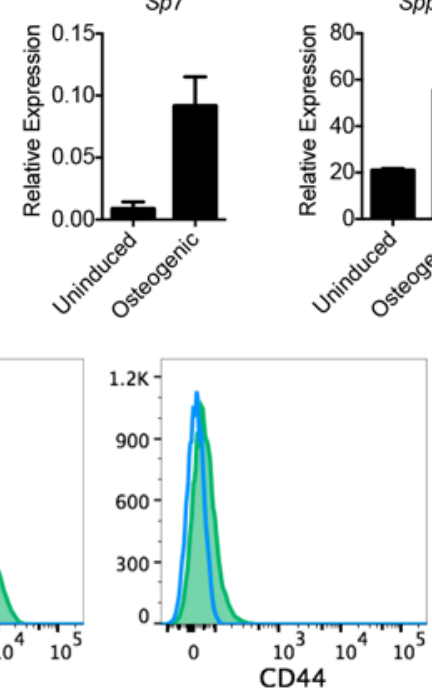
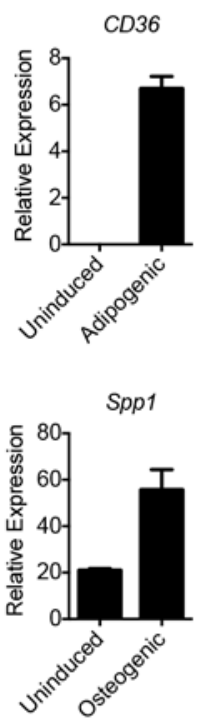

I Safranin $\mathrm{O}$

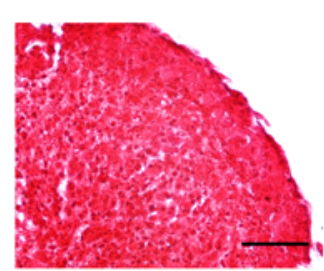

Acan
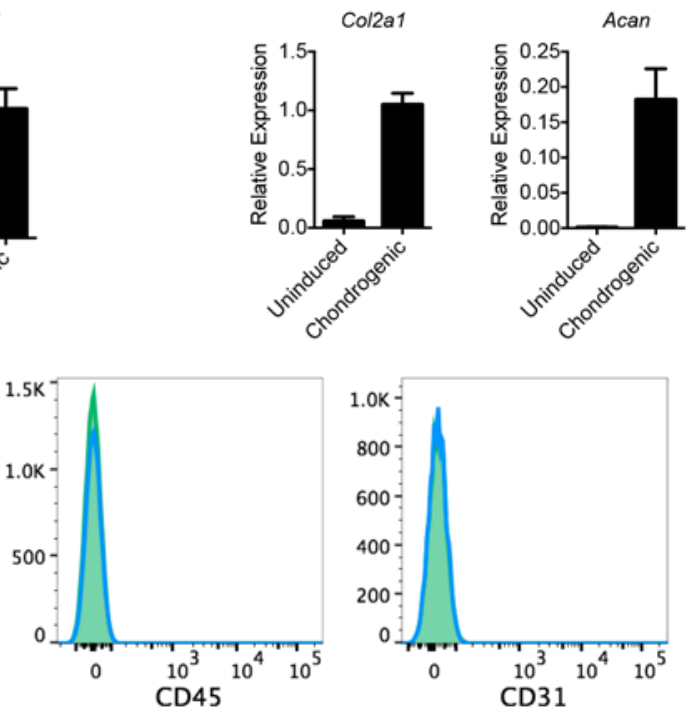
Figure 5. Aggrecan- and nuclear factor of activated T cells c1-expressing (NFATc1-expressing) cells at the enthesis are precursors of osteochondromas. (A) Fluorescent images of frozen sections of the knee joints from $N f a t c 7^{A g g C r e E R} N f a t c 2^{-1-} ; R o s a 26-m T m C^{f / /}$ (DKO;mTmG) mice 1 month after tamoxifen showing the majority of cells in osteochondromas are GFP+ (left panel). The image of higher magnification (right panel) reveals that cells without Cre recombination (white arrowheads) are also present. (B) Fluorescent images of knee joints from Nfatc1Cre;mTmG mice displaying Nfatc1 not expressed in growth plate (GP) cells. (C) Illustration showing tamoxifen-induced Cre-mediated recombination of the red fluorescence protein (RFP) reporter allele in Nfatc $1^{+}$cells from Nfatc1-CreERT2;Rosa26-RFPf// (Nfatc1CreER;RFP) mice leading to RFP expression (left panel). RFP fluorescence not detectable in GP cells 48 hours after tamoxifen pulse in 8-week-old Nfatc1CreER;RFP mice (right panel). (D) Fluorescent images of knee joints showing Nfatc1 expression in cells of the tibial medial collateral ligament (MCL) entheses and the perichondrium (PC)/periosteum (PO). (E) Fluorescent images of knee joints from AggCreER;mTmG and $D K O ; m T m G$ mice demonstrating cells in the periosteum adjacent to the MCL enthesis do not express Aggrecan (right panel, white arrowheads). GFP+ cells in left panel are in continuity with those at the MCL enthesis. OCM, osteochondroma. Images are representative of 4 or 5 mice per genotype except $\mathbf{C}$, in which 2 mice are used. (F) Schematics showing a subpopulation of cells at the tibial MCL enthesis expressing both Nfatc1 and Aggrecan. (G-I) Oil Red O, Alizarin Red S, and Safranin 0 staining, as well as quantitative PCR (qPCR) analyses, demonstrating adipogenic, osteogenic, and chondrogenic capacities of Aggrecan $^{+}$cells cultured ex vivo under lineage-specific differentiating conditions. (J) Flow cytometric analysis of stem cell (Sca1, CD90, and CD44), hematopoietic cell (CD45), and endothelial cell (CD31) markers in Aggrecan ${ }^{+}$cells from AggCreER; $m T m G$ mice. $n=3$ animals per group. Scale bars: $100 \mu \mathrm{m}$ (except in A, right panel, $25 \mu \mathrm{m}$ ).

Nfatc1-KO;mTmG or Nfatc2-KO;mTmG mice expressed higher levels of chondrogenic marker Comp and osteogenic marker Bglap (Figure 8F). These data suggest that Nfatc1 mainly inhibits the early stage of endochondral ossification, whereas Nfatc2 preferentially suppresses the late stage of endochondral ossification or osteogenesis.

\section{Discussion}

Here, we identify and characterize a new mechanism for osteochondromagenesis. Our results indicate that deficiency of the NFAT pathway causes osteochondroma formation at entheseal sites by enhancing the proliferation and osteochondral differentiation of entheseal progenitors. Depending on when during development NFAT expression is lost, and the number of $\mathrm{Nfatc1}$ and $\mathrm{Nfatc2}$ genes deleted, mice can develop the entire spectrum of human osteochondroma disease - from SO to mild HMO to severe HMO with growth retardation, skeletal deformation, and joint subluxation/dislocation. This observation indicates that osteochondroma formation is highly sensitive to the tumor suppressor activity of NFATs. However, the NFAT transcriptional factor family has been widely characterized as oncogenes in various tumors, and inhibition of NFAT activation using chemical or small molecular inhibitors, peptides, and compounds is being explored for anticancer therapy (21-24). Thus, our findings also provide a new perspective on the function of NFATs in tumorigenesis and suggest potential risks from clinical use of NFAT inhibitors.

Significant progress has been made toward understanding osteochondroma formation through clonal or sporadic inactivation of Ext1 in Collagen2-expressing cells in mice $(17,18)$. However, the factors that determine the severity of this disease, and the precise cellular origin of osteochondromas, remained unresolved. Our study supports the continuum model for tumor suppression (41), in which the severity of disease is sensitive to the dosage of loss of tumor suppressor genes. It is noteworthy that the role of NFATc1 and NFATc2 in human osteochondromas has not been characterized. Currently, it remains unclear that whether deficiency of NFAT signaling acts in the same pathway as that of EXT genes to cause osteochondromas. We did not detect any decrease of EXT gene expression in NFAT-deficient cells (data not shown), indicating that the NFAT pathway does not likely function as a direct transcriptional activator of the EXT genes. However, it is possible that NFAT acts downstream of EXT genes or is required for the expression of essential cofactors for EXT function. It should be highlighted that although mutations of EXT1 and/or EXT2 exist in the majority of HMO patients, the molecular understanding of how inactivation of these genes drives osteochondroma formation is limited. It has been hypothesized that mutations of EXT genes cause deficiency of the synthesis of heparin sulfate, which leads to disorganization of the extracellular matrix and abnormal diffusion of secreted cartilage- and bone-forming molecules, like BMPs, Wnts, TGF- $\beta$, and Ihh $(15,16)$. EXT mutations may thus perturb signaling pathways that result in the inactivation of osteochondroma tumor repressor genes, like NFATc1 and NFATc2. NFAT inactivation could occur at multiple levels encompassing transcriptional and translational mechanisms or via posttranslational modifications. Lastly, inactivation of NFATs could occur independently of EXT signaling and may be responsible for those HMO or SO patients lacking EXT disruptions. Future studies should carefully examine the expression and sequence of NFATs in human osteochondromas and characterize the potential crosstalk between EXT1/EXT2 and the NFAT pathway in osteochondroma formation. 
A
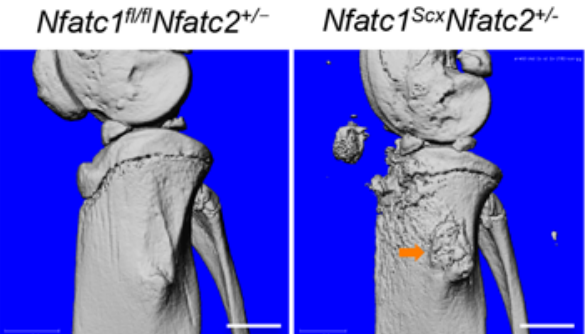

Nfatc $1^{\text {fl/fII }} \mathrm{Nfatc} 2^{-1-}$

Nfatc1 ${ }^{\text {Scx }}$ Nfatc2 $^{-1-}$
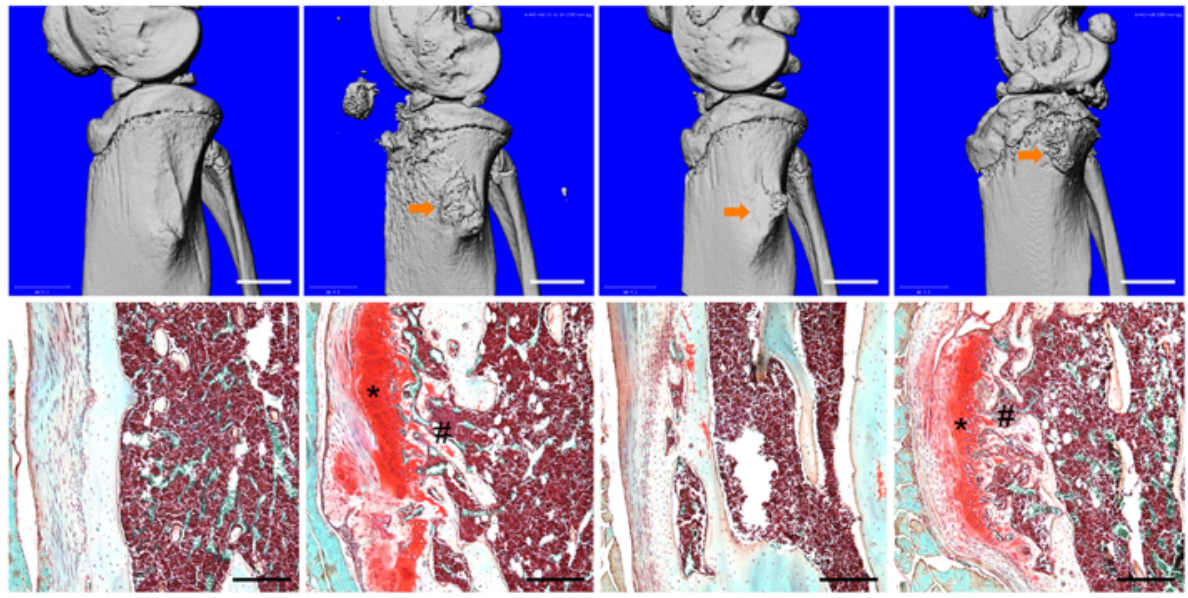

B
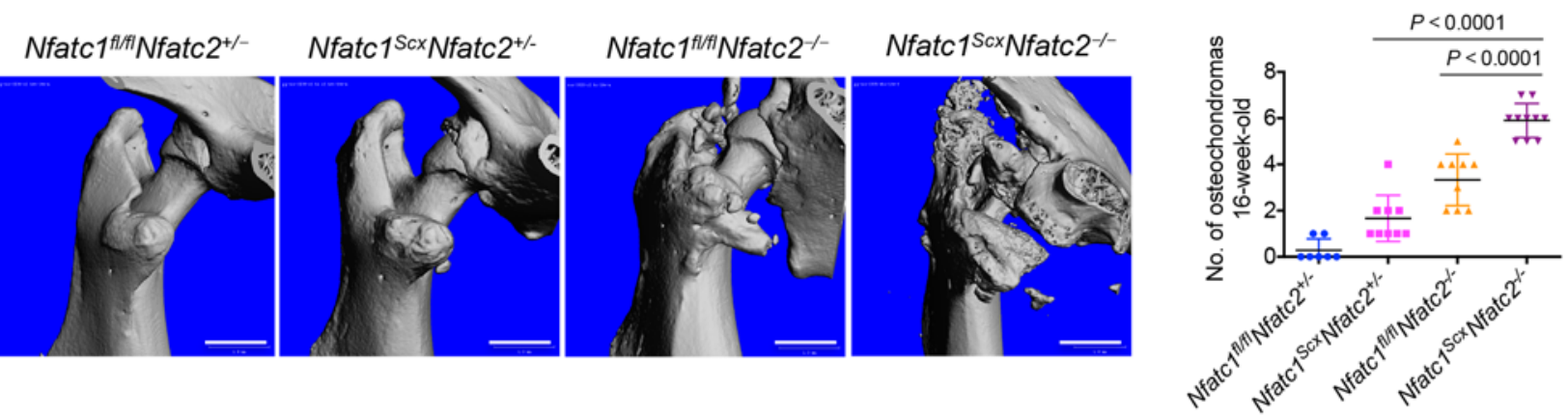

C

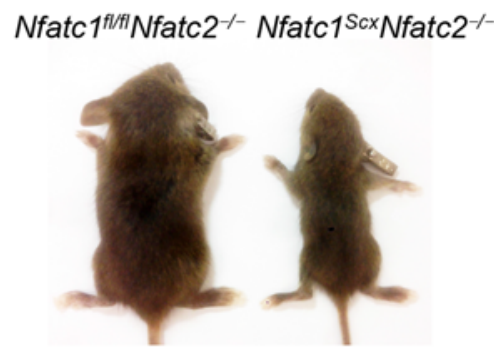

D

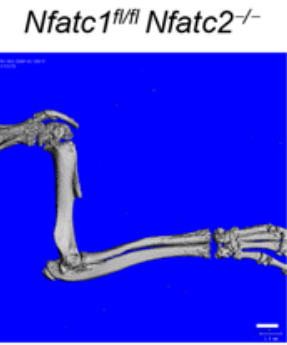

E

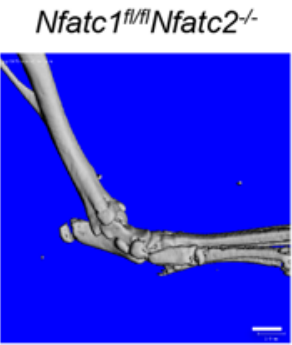

Nfatc1 ${ }^{\text {Scx }}$ Nfatc2 $^{-1}$

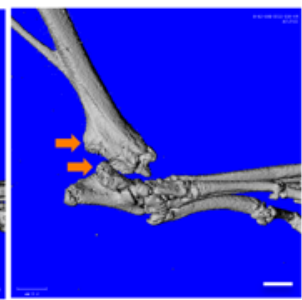

Figure 6. Nuclear factor of activated T cells c1 (NFATc1) deletion in Scleraxis-expressing entheseal progenitors results in osteochondromas. (A) $\mu$ CT images and safranin $\mathrm{O} /$ fast green stain showing osteochondroma formation at the tibial medial collateral ligament (MCL) entheses of 16-week-old

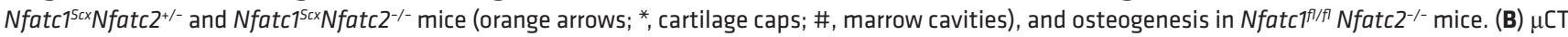

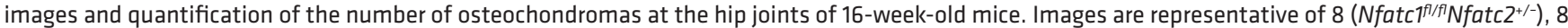

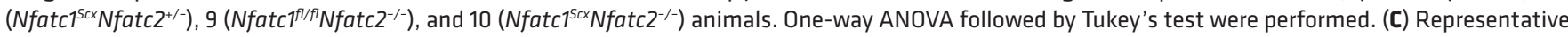

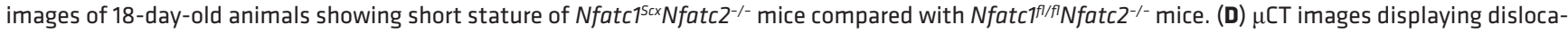

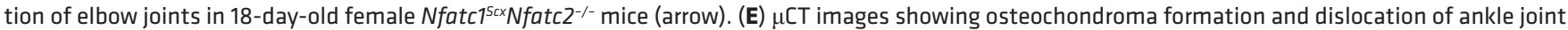

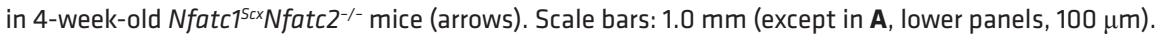

Current animal models of HMO by inactivating Ext1 in Collagen2-expressing cells suggest growth plate cells as the origin of the tumor (17-19). However, without the lineage tracing experiments, it is difficult to exclude that cells in the perichondrium and other sites, e.g., entheses, also expressing Collagen2, contribute to osteochondroma formation after inactivation of EXT1. Our study identified entheses as the location of osteochondroma formation and entheseal progenitor cells expressing Aggrecan, NFATc1, and Scx as the cellular origin of the tumor. With respect to the association of osteochondromas and entheses, Geschickter and Copeland, in the year of 1949, postulated that focal accumulations of cells with cartilaginous potential exist in all the points of tendinous insertion, and that continued stress and strains at these points might cause hyperplastic changes in these collections, which could result in osteochondromas (42). Several following case reports also showed that osteochondromas typically develop at the attachment sites of tendons 
Table 1. Summary of osteochondromas at the tibial medial collateral ligament (MCL) enthesis and subluxation/dislocation of ankle and elbow joints in mice with deletion of Nfatc1 in Scx-expressing entheseal progenitors on a WT or Nfatc2-deficient background

\begin{tabular}{|c|c|c|c|c|c|c|}
\hline \multirow{2}{*}{$\begin{array}{l}\text { 16-week-old } \\
\text { Genotypes }\end{array}$} & \multicolumn{4}{|c|}{ Osteochondromas at tibial MCL entheses } & \multicolumn{2}{|c|}{ Joint subluxation/dislocation } \\
\hline & Unilateral & Bilateral & Penetrance & $\%$ of bilateral & Ankle joints & Elbow joints \\
\hline Nfatc1 ${ }^{f l / f I} N$ fatc $^{+/-}$ & $0 / 8$ & $0 / 8$ & $0 \%$ & $0 \%$ & $0 / 8$ & $0 / 8$ \\
\hline Nfatc1 ${ }^{f l / f I} N$ fatc $2^{-/-}$ & $4 / 8$ & $2 / 8$ & $75 \%$ & $25 \%$ & $0 / 8$ & $0 / 8$ \\
\hline Nfatc1 ${ }^{S c x} N$ fatc $2^{+/-}$ & $3 / 9$ & $6 / 9$ & $100 \%$ & $67 \%$ & $0 / 9$ & $0 / 9$ \\
\hline Nfatc1 ${ }^{S c x} N$ fatc $2^{-/-}$ & $5 / 10$ & $2 / 10$ & $70 \%$ & $20 \%$ & $10 / 10$ & $5 / 10^{A}$ \\
\hline
\end{tabular}

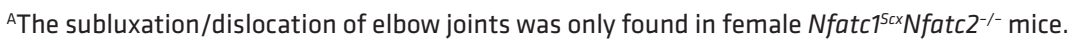

or ligaments (43-45). Consistent with our and other reports, a recent study showed that osteochondromas also outgrow from the ligamentous insertion sites in the cartilage tumor metachondromatosis after ablation of the protein tyrosine phosphatase SHP2 in fibroblasts by Fsp1-Cre (46). Furthermore, we think that localizing osteochondromas to the entheses may explain a current outstanding question about why osteochondromas always form near, but not directly adjacent to, the growth plate (47). Since there are numerous entheseal sites throughout the skeleton, it is easy to understand how individuals with the appropriate genetic lesion develop osteochondromas at many different anatomic sites.

Although NFAT family members are conserved and often display redundant functions, discrete biology among them has been appreciated, especially with regard to cell proliferation and cancer biology $(24,48)$. Our study extends the understanding of cell-specific functions for individual NFATs. Specifically, at the tibial MCL enthesis, NFATc1 maintains the normal proliferation or quiescence of entheseal progenitors and inhibits endochondral ossification, whereas NFATc2 appears to preferentially inhibit osteogenesis. However, osteochondral lesions around the hip joints of $N$ fatc2 $\mathrm{KO}$ mice (in this study and ref. 21) appear to contradict this mechanism. This potential discrepancy may be due to variability in the cellular composition of entheses (49), resulting in site-specific roles for NFATc2. In addition, recent studies also showed NFATc2 can directly inhibit osteoblast function and osteogenesis $(50,51)$, consistent with our finding.

There are some important limitations to our study. First of all, the detailed molecular understanding of how ablation of NFATc1 and NFATc2 affects the proliferation and osteochondral differentiation of entheseal progenitors remains unclear. Further study using RNA-sequencing or ChIP-sequencing will provide more comprehensive insight into the transcriptional regulation of this process. Secondly, for the lineage tracing during osteochondroma formation (Figure 5A), we only examined a single time point (1 month after tamoxifen). It is not clear whether the $\mathrm{GFP}^{+}$cells within the tumors result from the clonal expansion of either one or a few entheseal mesenchymal progenitors or, alternatively, whether all $\mathrm{GFP}^{+}$cells begin to divide once NFAT expression is lost. In addition, we did not carefully define how deletion of NFATs in entheseal mesenchymal progenitors affects the proliferation and differentiation of neighboring WT cells, which are also incorporated into the forming osteochondromas. Lastly, the global Nfatc2-null mice used in the present study display hyperproliferation of B- and T-lymphocytes and increased systemic levels of IL-4 and other inflammatory molecules $(51,52)$, which might affect osteochondroma formation. Conditional $N f a t c 2 \mathrm{KO}$ mice should be used in future studies to further explore the complementary and redundant roles of this transcriptional factor and NFATc1 in osteochondroma formation.

The molecular properties of the entheseal organ and its progenitors during development and in adult homeostasis remain poorly understood $(49,53)$. Given the findings in this study, we postulate that NFATc1 and NFATc2 are critical regulators of entheseal progenitors and that manipulation of this pathway could have therapeutic implications. In addition, our study suggests that Aggrecan expression identifies entheseal progenitors during development and adulthood in mice. A better understanding of these progenitors and methods to isolate them could lead to novel cellular therapies for enthesopathies. Future studies should also focus on determining whether NFAT gene dosage affects sarcomatous transformation of osteochondromas and the role of NFATs as tumor suppressors in other bone and cartilage neoplasms. Most importantly, tissue-specific regulation of NFAT activity and its upstream or downstream pathways should be explored as treatments for patients with skeletal diseases characterized by defective or exaggerated osteochondral growth. 
A

AggCreER ${ }^{T 2}$ Rosa26-mTmG $G^{f /+}$ (AggCreER;mTmG)

Nfatc1 ${ }^{\text {AggCreER Rosa26-mTmG }}{ }^{f /+}$ (Nfatc1-KO;mTmG)

AggCreER ${ }^{\text {T2Nfatc2 }}{ }^{-1-R o s a 26-m T m G}{ }^{f /++}$ (Nfatc2-KO;mTmG)

1 week after tamoxifen

Nfatc1 ${ }^{\text {AggCreER Nfatc2--Rosa26-mTmG }}{ }^{\text {fl/ }}$ (DKO;mTmG)

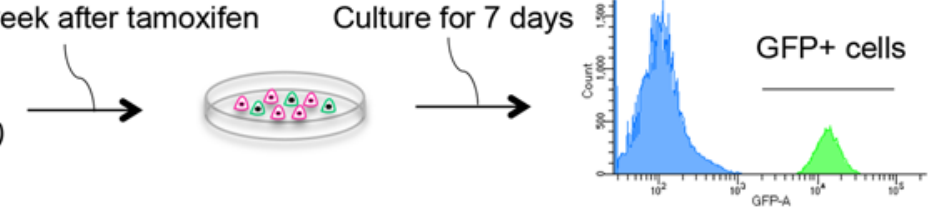

B

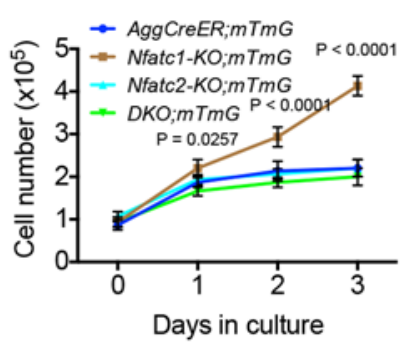

E

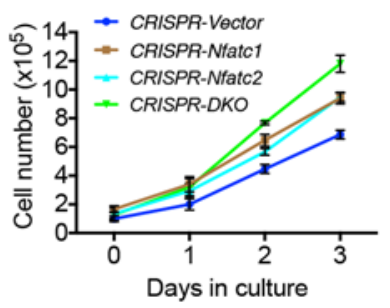

H

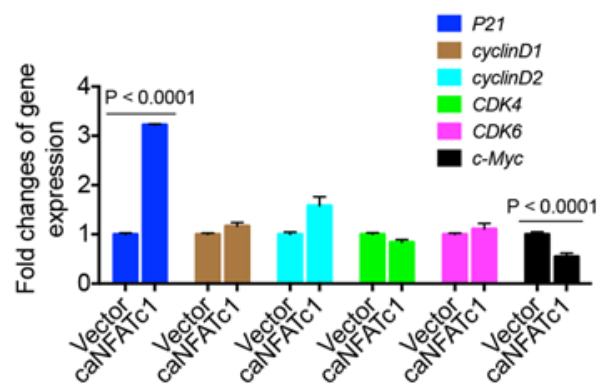

C

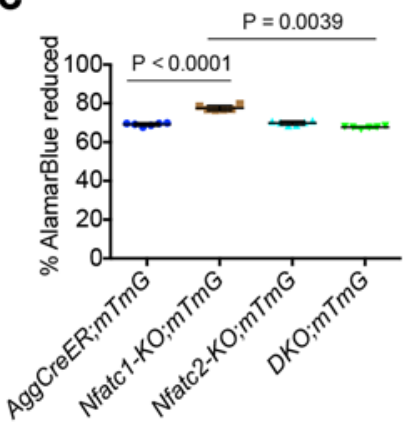

$\mathbf{F}$

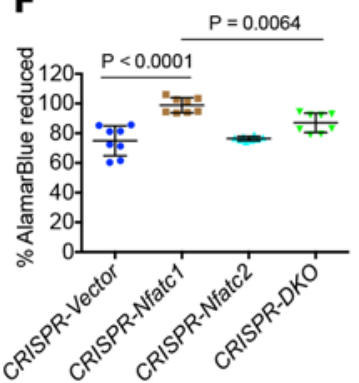

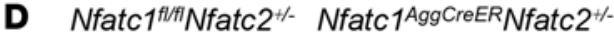
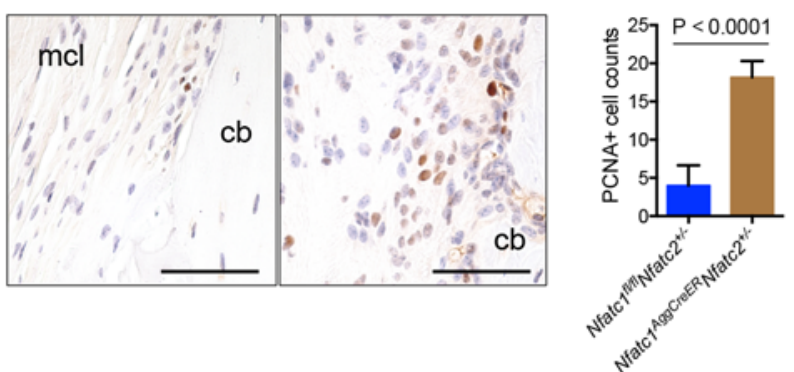

G
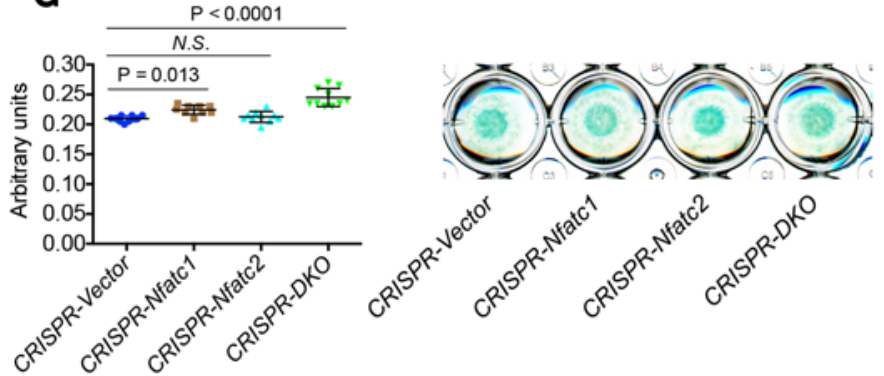

Figure 7. Ablation of nuclear factor of activated T cells c1 or c2 (NFATc1 or NFATc2) differentially affects proliferation of entheseal progenitors. (A) Schematic for isolating GFP+ ${ }^{+}$cells from the tibial medial collateral ligament (MCL) entheses of mice with indicated genotypes 1 week after tamoxifen. GFP+ cells were flow-sorted after 7 days of ex vivo expansion. (B and C) Cells from Nfatc1-KO;mTmG mice displaying increased proliferation as measured by cell count $(\mathbf{B}, n=3)$ and alamarBlue reduction (C, $n=6)$. (D) Representative anti-proliferating cell nuclear antigen (PCNA) IHC and quantification of PCNA+ cells at the tibial entheses of $N$ fatc $1^{f / f l} N$ fatc $2^{+/-}$and $N f a t c 1^{A g g C r e E R} N f a t c 2^{+/-}$mice 2 weeks after tamoxifen ( $n=3$ animals). Scale bars: $100 \mu$ m. (E) Cell proliferation assay of ATDC5 lines lacking Nfatc1, Nfatc2, or both under nondifferentiating conditions ( $n=3$ per sample). (F and G) Cell proliferation analyses by alamarBlue assay (F, $n=8$ per sample), micromass diameter (G, $n=10$ per sample), and representative micromass images after 2 weeks of micromass culture in chondrogenic media. Original magnification for the image in $\mathbf{G}: \times 1$. (H) Expression of cell cycle genes in ATDC5 cells overexpressing constitutively active NFATc1 (caNFATc1) by real-time PCR ( $n=3$ independent infections per sample). (I) Expression of $c-M y c$ and P21 genes in ATDC5 lines lacking Nfatc1, Nfatc2, or both ( $n=6$ per sample). Representative results of 3 independent experiments are presented. Two-way ANOVA followed by Tukey's (in B and E) or Sidak's (in H) tests were performed. $P$ values in C, F, G, and I were determined by 1-way ANOVA followed by Tukey's tests. Two-tailed Student's $t$ tests were performed for $\mathbf{D}$.

\section{Methods}

Mice. Nfatc1 ${ }^{f l f l}$ (54), Nfatc1-Cre (55), Nfatc1-CreERT2 (56), and Rosa26-RFP (56) mice have been previously described. Nfatc2-deficient, Aggrecan-CreERT2, and Scx-Cre mice were gifts from Anjana Rao (La Jolla Institute for Allergy and Immunology, La Jolla, California, USA), Benoit de Crombrugghe (The University of 
A

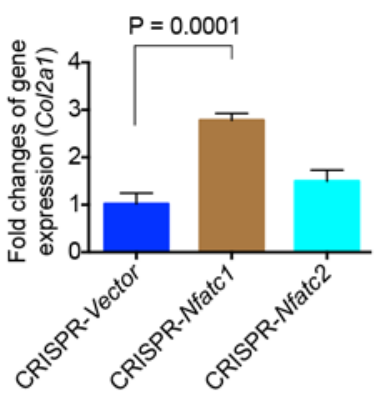

C

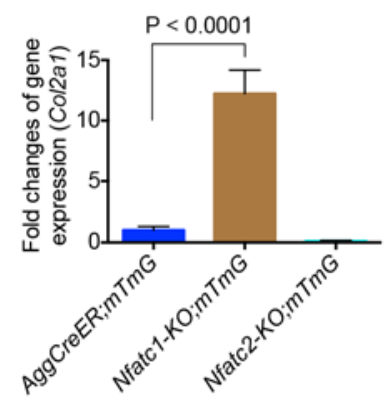

E

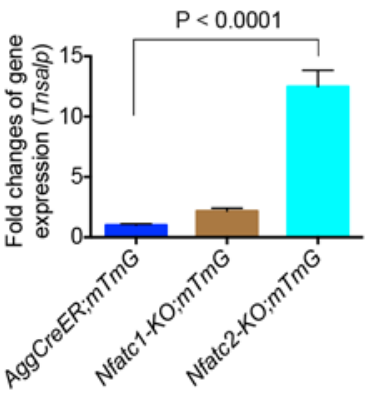

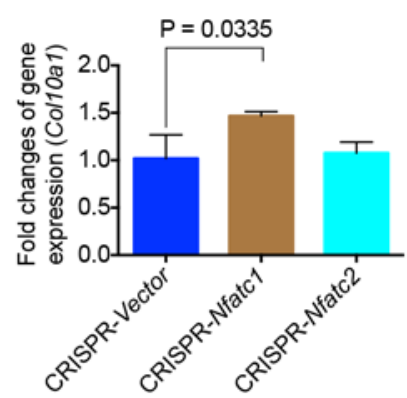
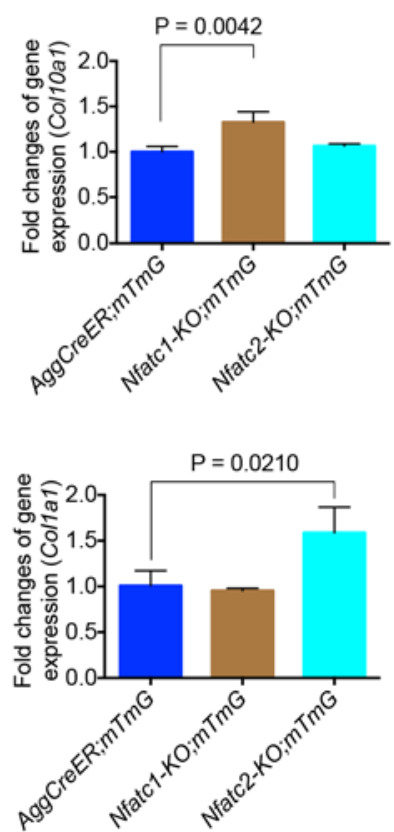

B

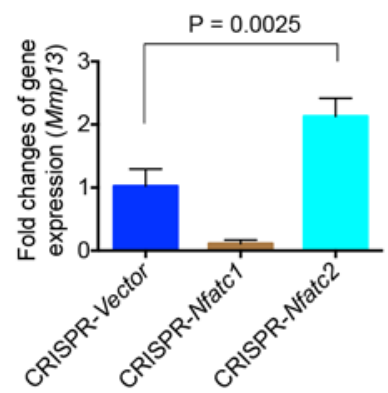

D

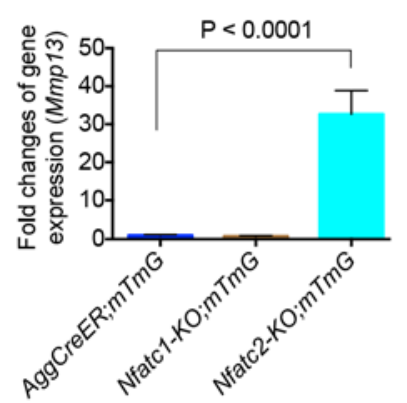

F

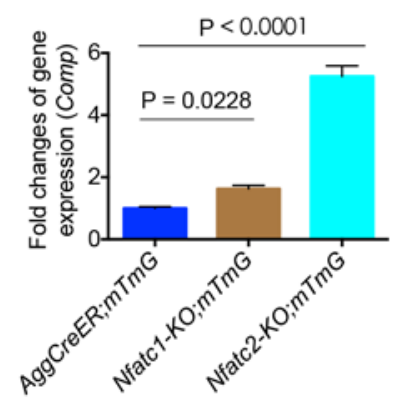

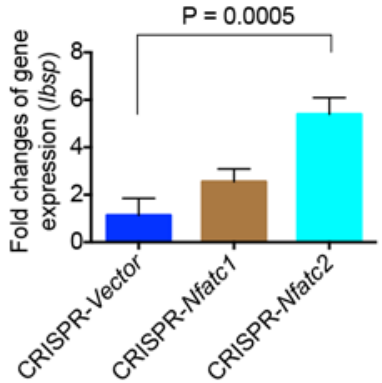
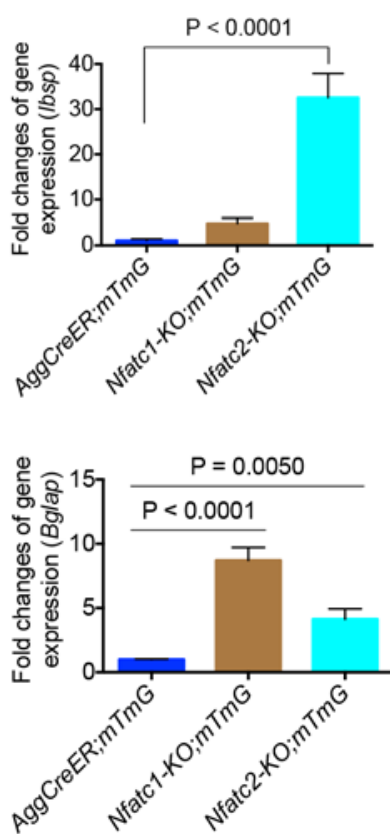

Figure 8. Nuclear factor of activated T cells c1 and c2 (NFATc1 and NFATc2) differentially repress genes characterizing endochondral ossification and osteogenesis. (A and B) Expression of Col2a1, Col10a1, Mmp13, and lbsp in ATDC5 cell lines with CRISP/Cas9-mediated deletion of Nfatc1 or Nfatc2 compared with Vector control after 2 weeks of micromass culture in chondrogenic media. (C and D) Expression of Col2a1, Col10a1, Mmp13, and Ibsp in GFP+ cells isolated from the tibial medial collateral ligament (MCL) entheses of AggCreER;mTmG, Nfatc1-KO;mTmG, and Nfatc2-KO;mTmG mice and cultured for 1 week ex vivo. (E and F) Expression of Tnsalp, Col1a1, Comp, and Bglap in the GFP+ cells from AggCreER;mTmG, Nfatc1-KO;mTmG, and Nfatc2-KO;mTmG mice. Gene expression was measured by real-time PCR using hypoxanthine phosphoribosyltransferase (Hprt) for normalization. $n=3$ per sample. Results are representative of 5 (A and B) or 3 (C-F) independent experiments. $P$ values were determined by 1-way ANOVA followed by Tukey's tests for multiple comparisons.

Texas MD Anderson Cancer Center, Houston, Texas, USA), and Cliff Tabin (Harvard Medical School, Boston, Massachusetts, USA), respectively. Prx1-Cre and Rosa26-mTmG $G^{A}$ mice were obtained from The Jackson Laboratory. Mice were maintained on either C57BL6, 129, or mixed background. All experimental animals were littermates except when otherwise indicated.

To induce Cre recombination in postnatal animals, Tamoxifen (T5648, Sigma-Aldrich; dissolved in ethanol and emulsified in sunflower oil, 1:9) was administered by i.p. injection for 5 consecutive days at 1 $\mathrm{mg} / 10 \mathrm{~g}$ body weight for adult mice and $0.5 \mathrm{mg} / 10 \mathrm{~g}$ body weight for 2 -week-old mice. Mice were housed in the animal facility of the Harvard T.H. Chan School of Public Health on a 12-hour light/dark cycle with ad libitum access to water and normal chow.

$\mu C T$. Ex vivo $\mu C T$ images were obtained using Scanco $\mu$ CT-35 (Scanco Medical) at $7 \mu \mathrm{m}$ resolution. Intact joints or limbs were scanned in a 7- or $12-\mathrm{mm}$ sample tube on medium (for 7-mm tube) or high (for 12-mm tube) resolution with default settings. The resulting images were then thresholded at 250 permille, and 3-dimensional reconstructions were generated. In vivo $\mu \mathrm{CT}$ images of live animals were acquired using an eXplore CT 120 scanner (GE Healthcare) with $50 \mu \mathrm{m}$ resolution. All scanning was performed using standard in vivo bone imaging protocol (360 views, 20-ms X-ray exposure time, penetration energy: $100 \mathrm{kV} / 50 \mathrm{~mA}$ ). Micro-CT images were reconstructed and visualized using Micro-View (GE Healthcare) and OsiriX. 
Histology, cartilage imaging, and IHC. Limb samples were fixed in 4\% PFA or 10\% neutral formalin for 24 hours at $4^{\circ} \mathrm{C}$. Postnatal skeletal tissues were decalcified for 2 weeks before embedding. Tissue sections ( 5 $\mu \mathrm{m})$ were stained with $\mathrm{H} \& \mathrm{E}$, Safranin O/fast green, or Alcian blue. Histopathologically, the typical osteochondromas were identified according to the WHO definition, including a cartilage cap and a medullary cavity continuous with that of underlying bone. Extracellular osteochondromas were defined as abnormal osteocartilaginous outgrowths without a direct attachment to bones but associated with ligaments, tendons or joint capsules.

Samples for lineage tracing were cryoprotected in $30 \%$ sucrose overnight at $4^{\circ} \mathrm{C}$ and then embedded in OCT and snap frozen. Cryosections $(8 \mu \mathrm{m})$ were counterstained using DRAQ5 and mounted in VECTASHIELD Antifade Mounting Medium (Vector Laboratories). Images were obtained using a Nikon TE2000-U confocal microscope or a KEYENCE BZ-X710 fluorescence microscope. For imaging the articular cartilage of the hip heads, intact bones were fixed and stained with DAPI dye $(10 \mu \mathrm{g} / \mathrm{ml})$ overnight and then scanned using the Zeiss LSM780 confocal laser scanning microscopy system at 1- or 2- $\mu$ m intervals from cartilage surface to a depth of $50 \mu \mathrm{m}$. IHC with anti-PCNA (1:5,000, AB29, Abcam) was performed using a M.O.M. kit (Vector Laboratories) as per the manufacturer's instructions. Mouse IgG2 $\alpha$ (BioLegend) was used as negative control.

Catwalk gait analysis. Gait analysis was performed using CatWalk XT 10.5 (Noldus Information Technology) as previously described $(57,58)$. Animals were allowed to walk voluntarily back and forth inside the walkway in the dark. Recorded runs with a steady walking speed (variation $<30 \%$ ) were accepted as compliant runs for paw print autoclassification as left front (LF), right front (RF), left hind (LH), and right hind $(\mathrm{RH})$ by the built-in software and classification was verified manually. Three to 6 runs were kept for calculation of gait parameters for every trial. Max contact max intensity represents the maximum intensity at max contact of a paw (ranging from 0-255). Stride length is the distance between successive placements of the same paw. Single stance is the duration (in seconds) of ground contact for a single hind paw.

In vitro analysis of Aggrecan-expressing entheseal cells. Tissues from the tibial MCL entheses were isolated under a dissecting microscope from 8-week-old AggCreER;mTmG, Nfatc1 ${ }^{A g g C r e E R} ; m T m G, A g g C r e E R ; N$ fatc2 $2^{--} ; m T m G$, and $N f a t c 1^{A g g C r e R} N f a t c 2^{---} ; m T m G$ mice 1 week after tamoxifen; cut into small pieces; and digested with $3 \mathrm{mg} / \mathrm{ml}$ collagenase type I (Worthington) and $4 \mathrm{mg} / \mathrm{ml}$ dispase (Roche Diagnostics) in serum-free media for 1 hour at $37^{\circ} \mathrm{C}$. After digestion, cells were passed through a $70-\mu \mathrm{m}$ filter and cultured for 7 days, and $\mathrm{GFP}^{+}$cells were sorted using FACSAria Fusion cell sorter (BD Biosciences). For CFU assays, single-cell suspensions of $\mathrm{GFP}^{+}$cells from WT mice were cultured in 6-well plates for 10 days. Colonies were stained and scored as described $(35,59)$. The ex vivo osteogenic, adipogenic, and chondrogenic differentiation potential of the Aggrecan-expressing entheseal cells was analyzed as described $(35,60,61)$.

Flow cytometry. The following antibodies were used: phycoerythrin-conjugated (PE-conjugated) antiSca1 (clone D7, BioLegend), anti-CD45 (clone 30-F11, eBioscience), and Rat IgG2a, $\kappa$ isotype control antibody (clone RTK2758, BioLegend), allophycocyanin (APC) anti-Thy-1/CD90.2 (clone 30-H12, eBioscience), anti-CD44 (clone IM7, BioLegend), anti-CD31 (clone 390, BioLegend), and Rat IgG2b, $\kappa$ isotype control antibody (clone RTK4530, BioLegend). Stained cells were analyzed on a FACSCanto II Flow Cytometer (BD Biosciences), and data analysis was performed using FlowJo software (Tree Star Inc.).

CRISPR/Cas9 lentivirus infection. Pairs of CRISPR guide RNA oligos (Nfatc1 single guide RNA [sgRNA] targeting TACGAGCTTCGGATCGAGGT on exon 3 and Nfatc2 sgRNA targeting GACTCGCATACCCGGATGAT on exon 2) were annealed and cloned into the BsmBI sites of lentiCRISPR V2-blasticidin and lentiCRISPR V2-puro (plasmid 52961, Addgene) vectors, respectively. CRISPR lentiviral plasmids and lentiviral packaging plasmids (pMDLg/pRRE, pRSV-Rev, and pMD2.G; Addgene) were transfected into 293T cells. Supernatants were harvested and filtered through a $0.45-\mu \mathrm{m}$ filter 2.5 days after transfection. ATDC5 cells (gift from Mary Goldring, Hospital for Special Surgery, New York, New York, USA) were infected with CRISPR lentivirus and selected with blasticidin (Invitrogen) (5 $\mu \mathrm{g} / \mathrm{ml})$ and puromycin (Clontech) $(4.5 \mu \mathrm{g} / \mathrm{ml})$ for 5 days and 7 days, respectively.

Retroviral infection. Control MSCV-GFP and constitutively nuclear caNFATc1 retroviral vectors were previously described (61). Retroviral constructs were transfected into Phoenix cells using Effectene Transfection Reagent (QIAGEN). Supernatants were harvested and filtered through a $0.45-\mu \mathrm{m}$ filter 48 hours after transfection. ATDC5 cells were infected by adding retroviral supernatant with $4 \mu \mathrm{g} / \mathrm{ml}$ polybrene for 72 hours, and $\mathrm{GFP}^{+}$cells were sorted for experiments. 
Cell proliferation analyses. Growth assays using sorted $\mathrm{GFP}^{+}$cell or CRISPR-infected cells were performed by trypsinizing and counting cells with a Countess Automated Cell Counter (Invitrogen) at indicated intervals. For cell viability assay, the same number of cells sorted using the BD FACSAria Fusion cell sorter was plated and cultured for 48-72 hours, and the AlamarBlue reagent (Thermo Scientific) was added and incubated for 4-8 hours. Fluorescence was measured with excitation wavelength at $545 \mathrm{~nm}$ and emission wavelength at $590 \mathrm{~nm}$ using the Synergy H1 Multi-Mode reader (Bio-Tek), and the percent reduction of AlamarBlue Reagent was calculated according to the manufacturer's instruction. Quantification of BrdU and cell cycle was performed using the APC-BrdU Flow Kit (BD Biosciences).

Real-time PCR and Western blotting. For real-time PCR, total RNA was isolated using either the Trizol reagent (Invitrogen) or the RNeasy mini kit (QIAGEN). RNA samples were treated with the RNase-Free DNase Set (QIAGEN) and equal amounts $(0.5-2 \mu \mathrm{g})$ were used for reverse transcriptase reaction using random primers (AffinityScript QPCR cDNA Synthesis Kit). PCR primer sequences are provided in Supplemental Table 2. Western blotting was performed as previously described (62) with the following antibodies: anti-NFATc1 (clone 7A6, BD Biosciences), anti-NFATc2 (clone 25A10.D6D2, Thermo Scientific), and anti-GAPDH (Cell Signaling Technology).

Statistics. All data are presented as mean \pm SD. Differences between 2 groups were evaluated by 2-tailed Student's $t$ test. Analyses of multiple groups were performed using 1-way ANOVA followed by Tukey's test for multiple comparisons. Two-way ANOVA, followed by Tukey's or Sidak's test for multiple comparisons, was performed as indicated. All analyses were performed using Prism 6.0 (GraphPad) or Excel (Microsoft). $P<0.05$ was considered significant.

Study approval. The Institutional Animal Care and Use Committee at Harvard Medical School approved all animal studies.

\section{Author contributions}

AOA and XG conceived the project and designed all experiments. XG performed all experiments. KT genotyped all mice. LH prepared the CRISPR/CAS9 lentiviruses. RAG contributed to the pathological diagnosis of mouse osteochondromas. FM helped for the FACS experiments. MZ helped to take cartilage imaging of whole bones. BZ (Albert Einstein College of Medicine of Yeshiva University) provided the Nfatc1-Cre mice. BZ (Chinese Academy of Sciences) prepared the samples of Nfatc1-CreER;RFP mice. XG and AOA analyzed the data and wrote the manuscript. JE contributed to the FACS staining and manuscript preparation.

\section{Acknowledgments}

We thank Gökhan S. Hotamisligil and Karen Inouye at Harvard T.H. Chan School of Public Health for help in acquiring in vivo $\mu \mathrm{CT}$ images; Matthew L. Warman at Boston Children's Hospital for suggestions to refine the manuscript; Yefu Li, Ruirui Shi, Kai Hu, and Jim McBride at Harvard School of Dental Medicine for technical assistance; and Xiaoyuan Huang at the Department of Pathology, Beijing Jishuitan Hospital and Peking University, for histological evaluation of mouse osteochondromas. The work was supported by NIH grants R01 AR060363 (to A.O. Aliprantis) and a Career Award for Medical Scientists from the Burroughs Wellcome Fund (to A.O. Aliprantis). X. Ge was fund by National Natural Science Foundation of China (project 81100767) and Ministry of Education of China (project 2092808).

Address correspondence to: Antonios O. Aliprantis, Merck Research Laboratories, 33 Avenue Louis Pasteur, Boston, Massachusetts 02115, USA. Phone: 617.992.3040; E-mail: aaliprantis@partners.org.

Antonios O. Aliprantis's present address is: Merck Research Laboratories, Boston, Massachusetts, USA.

1. Zhou S, et al. FGFR3 deficiency causes multiple chondroma-like lesions by upregulating hedgehog signaling. PLoS Genet. 2015;11(6):e1005214.

2. Bovee JV, Hogendoom PC, Wunder JS, Alman BA. Cartilage tumours and bone development: molecular pathology and possible therapeutic targets. Nat Rev Cancer. 2010;10(7):481-488.

3. Huegel J, Enomoto-Iwamoto M, Sgariglia F, Koyama E, Pacifici M. Heparanase stimulates chondrogenesis and is up-regulated in human ectopic cartilage: a mechanism possibly involved in hereditary multiple exostoses. Am J Pathol. 2015;185(6):1676-1685.

4. Khurana J, Abdul-Karim F, Bovee JVMG. Osteochondroma. In: Fletcher CDM, Bridge JA, Hogendoorn PCW, Mertens F, eds. WHO Classification of Tumours of Soft Tissue and Bone. 4th ed. Lyon, France: International Agency for Research on Cancer 
2013:234-236.

5. Kitsoulis P, et al. Osteochondromas: review of the clinical, radiological and pathological features. In Vivo. 2008;22(5):633-646

6. Murphey MD, Choi JJ, Kransdorf MJ, Flemming DJ, Gannon FH. Imaging of osteochondroma: variants and complications with radiologic-pathologic correlation. Radiographics. 2000;20(5):1407-1434.

7. Lim SC, Kim YS, Kim YS, Moon YR. Extraskeletal osteochondroma of the buttock. J Korean Med Sci. 2003;18(1):127-130.

8. Ueno T, Ansai S, Omi T, Kawana S. Extraskeletal osteochondroma arising on the plantar region. Case Rep Dermatol. 2011;3(2):147-150.

9. Estil JC Jr, Yeo ED, Kim HJ, Cho WT, Lee JJ. A large extraskeletal osteochondroma of the foot. J Foot Ankle Surg. 2013;52(5):663-665.

10. Panagopoulos L, et al. Rearrangement of chromosome bands 12q14 15 causing HMGA2-SOX5 gene fusion and HMGA2 expression in extraskeletal osteochondroma. Oncol Rep. 2015;34(2):577-584.

11. Jones KB, Pacifici M, Hilton MJ. Multiple hereditary exostoses (MHE): elucidating the pathogenesis of a rare skeletal disorder through interdisciplinary research. Connect Tissue Res. 2014;55(2):80-88.

12. Bovee JV, et al. EXT-mutation analysis and loss of heterozygosity in sporadic and hereditary osteochondromas and secondary chondrosarcomas. Am J Hum Genet. 1999;65(3):689-698.

13. Bovee JV. Multiple osteochondromas. Orphanet J Rare Dis. 2008;3:3.

14. Huegel J, et al. Perichondrium phenotype and border function are regulated by Ext1 and heparan sulfate in developing long bones: a mechanism likely deranged in Hereditary Multiple Exostoses. Dev Biol. 2013;377(1):100-112.

15. Huegel J, et al. Heparan sulfate in skeletal development, growth, and pathology: the case of hereditary multiple exostoses. Dev Dyn. 2013;242(9):1021-1032.

16. Jochmann K, Bachvarova V, Vortkamp A. Reprint of: Heparan sulfate as a regulator of endochondral ossification and osteochondroma development. Matrix Biol. 2014;35:239-247.

17. Matsumoto K, Irie F, Mackem S, Yamaguchi Y. A mouse model of chondrocyte-specific somatic mutation reveals a role for Ext1 loss of heterozygosity in multiple hereditary exostoses. Proc Natl Acad Sci U S A. 2010;107(24):10932-10937.

18. Jones KB, et al. A mouse model of osteochondromagenesis from clonal inactivation of Ext1 in chondrocytes. Proc Natl Acad Sci U S A. 2010;107(5):2054-2059.

19. Bovee JV. EXTra hit for mouse osteochondroma. Proc Natl Acad Sci U S A. 2010;107(5):1813-1814.

20. Zuntini M, et al. Genetic models of osteochondroma onset and neoplastic progression: evidence for mechanisms alternative to EXT genes inactivation. Oncogene. 2010;29(26):3827-3834.

21. Mancini M, Toker A. NFAT proteins: emerging roles in cancer progression. Nat Rev Cancer. 2009;9(11):810-820.

22. Müller MR, Rao A. NFAT, immunity and cancer: a transcription factor comes of age. Nat Rev Immunol. 2010;10(9):645-656

23. Qin JJ, et al. NFAT as cancer target: mission possible? Biochim Biophys Acta. 2014;1846(2):297-311.

24. Shou J, et al. Nuclear factor of activated T cells in cancer development and treatment. Cancer Lett. 2015;361(2):174-184.

25. Ranger AM, et al. The nuclear factor of activated T cells (NFAT) transcription factor NFATp (NFATc2) is a repressor of chondrogenesis. J Exp Med. 2000;191(1):9-22.

26. Szuhai K, et al. The NFATc2 gene is involved in a novel cloned translocation in a Ewing sarcoma variant that couples its function in immunology to oncology. Clin Cancer Res. 2009;15(7):2259-2268.

27. Arbajian E, et al. A benign vascular tumor with a new fusion gene: EWSR1-NFATC1 in hemangioma of the bone. Am J Surg Pathol. 2013;37(4):613-616.

28. Greenblatt MB, et al. NFATc1 and NFATc2 repress spontaneous osteoarthritis. Proc Natl Acad Sci U S A. 2013;110(49):19914-19919.

29. Buckland J. Osteoarthritis: Lack of Nfatc1 and Nfatc2: a new mouse model of OA. Nat Rev Rheumatol. 2014;10(1):4.

30. Peng SL, Gerth AJ, Ranger AM, Glimcher LH. NFATc1 and NFATc2 together control both T and B cell activation and differentiation. Immunity. 2001;14(1):13-20.

31. Graef IA, et al. Neurotrophins and netrins require calcineurin/NFAT signaling to stimulate outgrowth of embryonic axons. Cell. 2003;113(5):657-670.

32. Kim HK, Feng GS, Chen D, King PD, Kamiya N. Targeted disruption of Shp2 in chondrocytes leads to metachondromatosis with multiple cartilaginous protrusions. J Bone Miner Res. 2014;29(3):761-769.

33. Yang W, et al. Ptpn11 deletion in a novel progenitor causes metachondromatosis by inducing hedgehog signalling. Nature. 2013;499(7459):491-495.

34. Sugimoto $\mathrm{Y}$, et al. $\mathrm{Scx}^{+} / \mathrm{Sox} 9^{+}$progenitors contribute to the establishment of the junction between cartilage and tendon/ligament. Development. 2013;140(11):2280-2288.

35. Bi Y, et al. Identification of tendon stem/progenitor cells and the role of the extracellular matrix in their niche. Nat Med. 2007;13(10):1219-1227.

36. Stieber JR, Dormans JP. Manifestations of hereditary multiple exostoses. J Am Acad Orthop Surg. 2005;13(2):110-120.

37. Wuyts W, Schmale GA, Chansky HA, Raskind WH. Hereditary multiple osteochondromas. In: Pagon RA, et al. editors. GeneReviews. Seattle, Washington, USA: University of Washington; 2000, updated 2013.

38. Pedrini E, et al. Genotype-phenotype correlation study in 529 patients with multiple hereditary exostoses: identification of "protective" and "risk" factors. J Bone Joint Surg Am. 2011;93(24):2294-2302.

39. Guo XL, Deng Y, Liu HG. Clinical characteristics of hereditary multiple exostoses: a retrospective study of mainland chinese cases in recent 23 years. J Huazhong Univ Sci Technolog Med Sci. 2014;34(1):42-50.

40. Atsumi T, Miwa Y, Kimata K, Ikawa Y. A chondrogenic cell line derived from a differentiating culture of AT805 teratocarcinoma cells. Cell Differ Dev. 1990;30(2):109-116.

41. Berger AH, Knudson AG, Pandolfi PP. A continuum model for tumour suppression. Nature. 2011;476(7359):163-169.

42. Geschickter CF, Copeland MM. Tumors Of The Bone. 3rd ed. Philadelphia, Pennsylvania, USA: J.B. Lippincott Co.; 1949.

43. Gokay H, Bucy PC. Osteochondroma of the lumbar spine; report of a case. J Neurosurg. 1955;12(1):72-78.

44. Ramon Y, Lerner MA, Leventon G. Osteochondroma of the mandibular condyle. Report of a case. Oral Surg Oral Med Oral Pathol. 1964;17:16-21.

45. Nakamura T, Kojima T. Soft tissue osteochondroma originating from FDS tendon insertion. J Hand Surg Eur Vol. 
2010;35(9):764-766.

46. Bowen ME, Ayturk UM, Kurek KC, Yang W, Warman ML. SHP2 regulates chondrocyte terminal differentiation, growth plate architecture and skeletal cell fates. PLoS Genet. 2014;10(5):e1004364.

47. Pacifici M. Pathogenesis of MHE: Recent advances and prospects for therapies. Semin Arthritis Rheum. 2013;42(5):547.

48. Robbs BK, Cruz AL, Werneck MB, Mognol GP, Viola JP. Dual roles for NFAT transcription factor genes as oncogenes and tumor suppressors. Mol Cell Biol. 2008;28(23):7168-7181.

49. Benjamin M, et al. Where tendons and ligaments meet bone: attachment sites ('entheses') in relation to exercise and/or mechanical load. J Anat. 2006;208(4):471-490.

50. Zanotti S, Canalis E. Activation of Nfatc2 in osteoblasts causes osteopenia. J Cell Physiol. 2015;230(7):1689-1695.

51. Zanotti S, Smerdel-Ramoya A, Canalis E. Nuclear factor of activated T-cells (NFAT)C2 inhibits Notch receptor signaling in osteoblasts. J Biol Chem. 2013;288(1):624-632.

52. Hodge MR, Ranger AM, Charles de la Brousse F, Hoey T, Grusby MJ, Glimcher LH. Hyperproliferation and dysregulation of IL-4 expression in NF-ATp-deficient mice. Immunity. 1996;4(4):397-405.

53. Apostolakos J, et al. The enthesis: a review of the tendon-to-bone insertion. Muscles Ligaments Tendons J. 2014;4(3):333-342.

54. Aliprantis AO, et al. NFATc1 in mice represses osteoprotegerin during osteoclastogenesis and dissociates systemic osteopenia from inflammation in cherubism. J Clin Invest. 2008;118(11):3775-3789.

55. Wu B, et al. Endocardial cells form the coronary arteries by angiogenesis through myocardial-endocardial VEGF signaling. Cell. 2012;151(5):1083-1096.

56. Tian X, et al. Vessel formation. De novo formation of a distinct coronary vascular population in neonatal heart. Science 2014;345(6192):90-94

57. Fu SC, Cheuk YC, Hung LK, Chan KM. Limb Idleness Index (LII): a novel measurement of pain in a rat model of osteoarthritis. Osteoarthritis Cartilage. 2012;20(11):1409-1416.

58. Parvathy SS, Masocha W. Gait analysis of C57BL/6 mice with complete Freund's adjuvant-induced arthritis using the CatWalk system. BMC Musculoskelet Disord. 2013;14:14.

59. Nagatomo K, et al. Stem cell properties of human periodontal ligament cells. J Periodontal Res. 2006;41(4):303-310

60. Seo BM, et al. Investigation of multipotent postnatal stem cells from human periodontal ligament. Lancet. 2004;364(9429):149-155

61. Horsley V, Aliprantis AO, Polak L, Glimcher LH, Fuchs E. NFATc1 balances quiescence and proliferation of skin stem cells. Cell. 2008;132(2):299-310

62. Ge X, et al. Role of Wnt-5A in interleukin-1beta-induced matrix metalloproteinase expression in rabbit temporomandibular joint condylar chondrocytes. Arthritis Rheum. 2009;60(9):2714-2722. 